TRANSACTIONS OF THE

AMERICAN MATHEMATICAL SOCIETY

Volume 355, Number 8, Pages 3405-3432

S 0002-9947(03)03275-6

Article electronically published on April 11, 2003

\title{
LOCAL SOLVABILITY AND HYPOELLIPTICITY FOR SEMILINEAR ANISOTROPIC PARTIAL DIFFERENTIAL EQUATIONS
}

\author{
GIUSEPPE DE DONNO AND ALESSANDRO OLIARO
}

\begin{abstract}
We propose a unified approach, based on methods from microlocal analysis, for characterizing the local solvability and hypoellipticity in $C^{\infty}$ and Gevrey $G^{\sigma}$ classes of 2-variable semilinear anisotropic partial differential operators with multiple characteristics. The conditions imposed on the lower-order terms of the linear part of the operator are optimal.
\end{abstract}

\section{INTRODUCTION}

We consider a class of semilinear anisotropic equations with multiple characteristics in two variables $(x, y)$ belonging to the set $\Omega:=\left\{\sqrt{x^{2}+y^{2}}<\delta\right\}, \delta$ sufficiently small, of the form

$$
P\left(x, y, D_{x}, D_{y}\right) u+\left.F\left(x, y, \partial_{x}^{l} \partial_{y}^{j} u\right)\right|_{\mid \frac{m}{d}+j<m-t}=\mu f(x, y),
$$

where the linear part is given by

$$
P\left(x, y, D_{x}, D_{y}\right)=D_{y}^{m}-b_{0}(x, y) D_{x}^{d}+\sum_{m-t \leq l \frac{m}{d}+j<m} a_{l j}(x, y) D_{x}^{l} D_{y}^{j},
$$

with $m, d, j, l \in \mathbb{Z}_{+}, 0<t<\frac{1}{2}, \mu$ sufficiently small, $D_{x}=-i \frac{\partial}{\partial x}, D_{y}=-i \frac{\partial}{\partial y}$; we shall also say that $l \frac{m}{d}+j$ is the anisotropic order of $D_{x}^{l} D_{y}^{j}$, and so the nonlinearity involves derivatives of anisotropic order less than $m-t$. Our main aim is to propose a unified approach for a complete analysis of the influence of the lower-order terms of (1.2) on the solvability and hypoellipticity of (1.1) in the $C^{\infty}$ category and in the Gevrey spaces $G^{\sigma}$ beyond the critical index $m /(m-1)$. The arguments in our proofs are based mainly on microlocal tools: pseudo-differential operators, wave front sets, allowing relevant simplifications in the study, and $S_{\rho, \delta}^{m}$ techniques.

Some papers have been devoted to the study of this kind of problem; see, for example, Hounie-Santiago [HS and Gramchev-Popivanov GP on the local solvability of semilinear partial differential equations in the case of simple characteristics, Gramchev-Rodino GR about Gevrey solvability for equations with multiple characteristics (see also Spagnolo [SP] and Kajitani-Spagnolo [KS]), and Garello [G] regarding the inhomogeneous elliptic case; see also Šananin [S] on the $C^{\infty}$ local solvability of equations of quasi-principal type and Lorenz [LO] regarding anisotropic operators with characteristics of constant multiplicity.

Received by the editors February 7, 2001 and, in revised form, October 8, 2002.

2000 Mathematics Subject Classification. Primary 35S05.

(C)2003 American Mathematical Society 
We consider an $F$ that is $C^{\infty}$ and nonlinear, and we assume that the coefficients in (1.2) are $C^{\infty}$. We always suppose that

$$
d<m, \quad \Re b_{0}(0,0) \neq 0, \quad F(x, y, 0)=0 .
$$

We recall that the nonzero requirement on $b_{0}$ is an invariant nondegeneracy condition, usually required in the study of the local solvability and hypoellipticity of the linear operator (1.2) in $C^{\infty}$ and in the Gevrey classes $G^{\sigma}, \sigma>\frac{m}{m-1}$; see for example Liess-Rodino [LR2], De Donno-Rodino [DR2], in which Gevrey hypoellipticity for PDEs with high multiplicity is proved. Let us also observe that if $\Im b_{0}(x, y) \neq 0$, then the operator is quasi-elliptic; the results of hypoellipticity and local solvability are well known in this case. Regarding $G^{\sigma}$ data, see for example Marcolongo-Oliaro [M], in which the local solvability is proved in the $n$-dimensional case and under hypotheses on the quasi-principal symbol; in the present paper we admit less regular data $f(x, y)$ with respect to the case studied in [MO], but we add hypotheses on the lower-order terms. In this frame it will be convenient to use the Sobolev anisotropic space $H_{\frac{1}{q}}^{s}, q \geq 1$, defined by

$$
\|f\|_{H_{\frac{1}{q}}^{s}}:=\int\left(1+|\xi|^{\frac{2}{q}}+|\eta|^{2}\right)^{s}|\widehat{f}(\xi, \eta)|^{2} d \xi d \eta<\infty
$$

$\widehat{f}(\xi, \eta)$ being the Fourier transform of $f(x, y)$. For $s>\frac{1+q}{2}, H_{\frac{1}{q}}^{s}$ is an algebra; cf. the inhomogeneous Schauder estimates and Garello [G, Proposition 2.5]. Moreover, we define the anisotropic characteristic manifold

$$
\Sigma_{\delta}:=\left\{(x, y, \xi, \eta) \in \Omega \times \mathbb{R}^{2} \backslash\{0\}, \eta^{m}-\Re b_{0} \xi^{d}=0\right\}
$$

We recall the definition of the Gevrey anisotropic space $G^{\left(q_{1}, q_{2}\right)}(\Omega)$.

Definition 1.1. Let $q_{1}>1, q_{2}>1$. We denote by $G^{\left(q_{1}, q_{2}\right)}(\Omega)$ the set of all the functions $f \in C^{\infty}(\Omega)$ such that the following condition holds: for every compact $K \subset \Omega$ there exists a positive constant $C_{K}$ such that $\sup _{K}\left|\partial_{x}^{l} \partial_{y}^{j} f(x, y)\right| \leq$ $C_{K}^{l+j+1}(l !)^{q_{1}}(j !)^{q_{2}}$ for every $l, j \in \mathbb{Z}_{+}$.

As usual, $G_{0}^{\left(q_{1}, q_{2}\right)}(\Omega)$ is the set of all the functions in $G^{\left(q_{1}, q_{2}\right)}(\Omega)$ with compact support in $\Omega$.

Let us state the main results.

Theorem 1.1. Let $\left(l^{*}, j^{*}\right) \in \mathbb{Z}_{+}^{2}$ be the unique couple in $\mathbb{Z}_{+}^{2}$ having anisotropic order $\frac{k^{*}}{d}=l^{*} \frac{m}{d}+j^{*}$, with $d\left(m-\frac{1}{2}\right)<k^{*}=m l^{*}+d j^{*}<d m$. We assume that for $(x, y, \xi, \eta) \in \Sigma_{\delta}$ :

i) $\Im a_{l^{*} j^{*}}(x, y) \neq 0$,

ii) for all $(l, j)$ such that $d j+m l>k^{*}$,

$$
\Im a_{l^{*} j^{*}}(x, y) \Im a_{l j}(x, y) \xi^{l+l^{*}} \eta^{j+j^{*}} \geq 0,
$$

iii) $\Im a_{l^{*} j^{*}}(x, y) \Im b_{0}(x, y) \xi^{d+l^{*}} \eta^{j^{*}} \leq 0$. 
Choose and fix $t=m-\frac{k^{*}}{d}$ and $s>\frac{1}{2} \frac{d+m}{d}+\frac{k^{*}}{d}$. Then we can find $\delta_{0}>0$, depending on $P$ and $s$, such that for every $f \in H_{\frac{d}{m}}^{s}(\Omega)$ with compact support in $\Omega$ the equation (1.1) admits a solution $u \in H_{\frac{d}{m}}^{s+m-r^{*}}$ with $r^{*}=m-\frac{k^{*}}{d}$, provided $\mu=1$ if $F \equiv 0$ and $\mu\|f\|_{H_{\frac{d}{m}}^{s}}<\mu_{0}$ for some $0<\mu_{0} \ll 1$ depending on the nonlinear term $F$ if $F \not \equiv 0$. Finally, the linear operator $P$ in 1.2 is $C^{\infty}(\Omega)$ hypoelliptic, and if its coefficients are analytic, $P$ is $G^{\sigma}(\Omega)$ hypoelliptic, $\sigma \geq \frac{d}{k^{*}-d(m-1)}$, i.e., if $u$ is a distribution in $\Omega$ such that $P u \in G^{\sigma}(\Omega)$, then $u \in G^{\sigma}(\Omega)$.

Remark 1.1. The conditions (1.5) in Theorem 1.1 could be illustrated in a simpler way, observing that actually one estimates the imaginary part of the symbol $\sum_{\frac{k^{*}}{d} \leq l \frac{m}{d}+j<m} a_{l j}(x, y) \xi^{l} \eta^{j}$ in the corresponding operator (1.2) on the quasi-conic characteristic set $\eta^{m}-\Re b_{0} \xi^{d}=0$, e.g., substituting $\xi=\left(\Re b_{0}\right)^{-\frac{1}{d}} \eta^{\frac{m}{d}}$, the condition (1.5) reads

$$
\left|\Im\left(\sum_{\frac{k^{*}}{d} \leq l \frac{m}{d}+j<m} a_{l j}\left(\Re b_{0}\right)^{-\frac{l}{d}} \eta^{\frac{l m}{d}} \eta^{j}\right)\right| \geq C|\eta|^{\frac{k^{*}}{d}}, \quad \eta \gg 1,
$$

with $k^{*}=m l^{*}+d j^{*}$. This clarifies, at least intuitively, the loss of derivatives $\frac{k^{*}}{d}$ in Theorem 1.1].

Remark 1.2. It is always possible to rephrase the previous assumptions in Theorem 1.1 directly on the coefficients of $P$. For example, if $\Re b_{0}>0$ and $m, d$ are odd, the conditions $i), i i), i i i)$ are respectively equivalent to:

$\left.i^{\prime}\right) \Im a_{l^{*} j^{*}}(x, y)>0(<0)$;

$\left.i i^{\prime}\right)$ for all $(l, j)$ such that $d j+m l>k^{*}=d j^{*}+m l^{*}, \Im a_{l j}(x, y) \geq 0(\leq 0)$ for $j+j^{*}$ and $l+l^{*}$ both even or both odd, and $\Im a_{l j}(x, y) \equiv 0$ otherwise;

$\left.i i i^{\prime}\right) \Im b_{0}(x, y) \leq 0(\geq 0)$ for $j^{*}$ and $d+l^{*}$ both even or both odd, and $\Im b_{0}(x, y) \equiv$ 0 otherwise.

In the picture on the next page, which resembles the Newton polygon pictures, we show the geometrical meaning of hypothesis ii) in Theorem 1.1 (or equivalently assumption $i i^{\prime}$ ) in Remark [1.2). We consider the operator of order $m=9$ with $d=7$ :

$$
D_{y}^{9}-\left(1-i y^{2 k}\right) D_{x}^{7}+y^{h} D_{x}^{3} D_{y}^{5}+i D_{x}^{6} D_{y}+\sum_{\frac{9}{7} l+j<\frac{61}{7}} a_{l j}(x, y) D_{x}^{l} D_{y}^{j},
$$

is $C^{\infty}$ and Gevrey hypoelliptic and $C^{\infty}$ solvable by Theorem 1.1 .

We want to study now the case in which hypothesis $i$ ) in Theorem 1.1 is not satisfied: the basic idea is to refer to the Gevrey classes and transform the operator $P$ in (1.2) into another operator that satisfies it. To this aim, we introduce the anisotropic Gevrey-Sobolev spaces $\mathbb{H}_{\tau, q, r}^{s, \psi}(\mathbb{R} \times(-\delta, \delta))$, defined as the set of all $L^{2}$ functions for which

$$
\|f\|_{\mathbb{H}_{\tau, q, r}^{s, \psi}}:=\left\|e^{\tau \psi\left(y, D_{x}\right)} f\right\|_{H_{\frac{1}{q}}^{s}}<+\infty
$$

where $q \geq 1$ is the Gevrey order, $s>0$ the Sobolev index, and we take $r \in(0,1)$, $\tau>0 ; \psi=\psi(y, \xi)$ is a nonnegative function belonging to the Hörmander class $S_{1,0}^{\frac{r}{q}}((-\delta, \delta) \times \mathbb{R})$. 


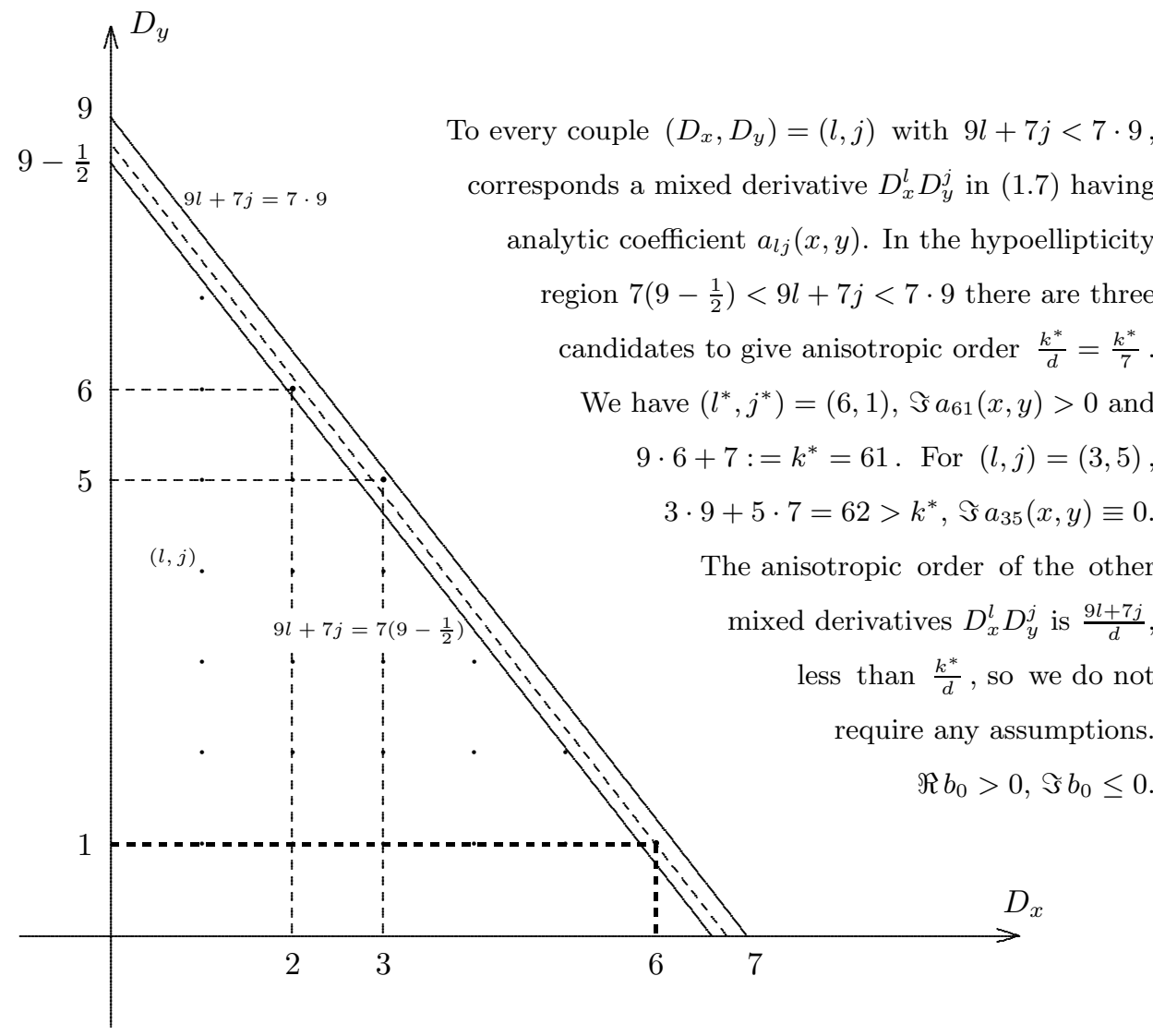

Theorem 1.2. In the equation (1.1) let the datum $f$ be in $G_{0}^{\left(\frac{m}{d r}, q_{2}\right)}(\Omega), r \in\left(\frac{1}{2}, 1\right)$, $q_{2}>1$. Fix $t>1-r$ and assume that the coefficients of $P$ in (1.2) are analytic and for $(x, y, \xi, \eta) \in \Sigma_{\delta}$ one of the following conditions holds:

[a] $\Im a_{l j}(x, y) \xi^{l} \eta^{j+m-1} \leq 0(\geq 0)$ for $d j+m l>(m-t) d$, and, moreover, $\Im b_{0}(x, y) \xi^{d} \eta^{m-1} \geq 0(\leq 0)$;

[b] $\Im a_{l j}(x, y)(\operatorname{sign} \xi) \xi^{l} \eta^{j+m-1} \leq 0(\geq 0)$ for $d j+m l>(m-t) d$, and $\Im b_{0}(x, y)$ $(\operatorname{sign} \xi) \xi^{d} \eta^{m-1} \geq 0(\leq 0)$.

Assume moreover that the nonlinear term $F$ is analytic with respect to $(x, y)$, entire with respect to $\partial_{x}^{l} \partial_{y}^{j} u$, and (1.3) holds. Then, fixing $\psi$ and taking s large, we can find $\delta_{0}>0$, depending on $P$ and $s$, such that for every $f \in \mathbb{H}_{\tau, \frac{m}{d}, r}^{s, \psi}(\Omega)$ with compact support in $\Omega$ the semilinear equation (1.1) admits a solution $u \in \mathbb{H}_{\tau, \frac{m}{d}, r}^{s+m-(1-r), \psi}(\Omega)$ provided $\mu=1$ if $F \equiv 0$, and $\mu\|f\|_{\mathbb{H}_{\tau, \frac{m}{d}, r}^{s, \psi}}<\mu_{0}$ for some $0<\mu_{0} \ll 1$ depending on the nonlinear term $F$ if $F \not \equiv 0$.

The study of the weakly hyperbolic equation (1.1) resembles the study of degenerate parabolic equations, in that under suitable hypotheses equation (1.1) behaves like them in regard to local solvability and hypoellipticity in the $C^{\infty}$ category and in 
some Gevrey spaces $G^{\sigma}, \sigma>\frac{m}{m-2}$. See, for example, Gramchev-Popivanov-Yoshino GPY Section 3].

In Theorem 1.2 we require that the nonlinearity is analytic with respect to $\left(x, y, \partial_{x}^{l} \partial_{y}^{j} u\right)$. One encounters highly nontrivial difficulties in getting hard analysis type estimates on composition of nonanalytic Gevrey nonlinearities, and in the paper Gramchev-Rodino GR different Gevrey norms involving power series of finite Sobolev-type norms are used. A possible analogue of Theorem 1.2 could be proved in the anisotropic case for nonanalytic Gevrey nonlinearities, and this will be the subject of a future paper.

We point out that in hypotheses [a] and [b] of Theorem 1.2 the exponent $m-1$ plays the role of $j^{*}$ in assumptions $i i$ ) and $\left.i i i\right)$ of Theorem 1.1. In fact, for a suitable fixed $\psi(y, \xi) \in S_{1,0}^{\frac{r}{q}}((-\delta, \delta) \times \mathbb{R})$, the operator

$$
\widetilde{P}:=e^{\tau \psi\left(y, D_{x}\right)} P\left(x, y, D_{x}, D_{y}\right) e^{-\tau \psi\left(y, D_{x}\right)}
$$

contains all the terms of $P$ and an additional pseudo-differential term

$$
N_{r}(x, y)\left|D_{x}\right|^{\frac{d}{m} r} D_{y}^{m-1},
$$

where $N_{r}(x, y)$ satisfies condition $\left.i\right)$ in Theorem 1.1 .

Observe that for the existence of $l, j \in \mathbb{Z}_{+}$such that $m-t \leq l \frac{m}{d}+j<m, t<\frac{1}{2}$, we have to require $m \geq 4$. The theorem applies, of course, also in the case when the assumption on the $a_{l j}$ is empty; in this case we recapture the result of [MO].

Passing to the standard isotropic Gevrey classes $G^{\sigma}(\Omega)$, defined by the estimates

$$
\sup _{K}\left|\partial_{x}^{l} \partial_{y}^{j} f(x, y)\right| \leq C^{l+j+1}(l ! j !)^{\sigma}, \quad K \subset \subset \Omega,
$$

we may conclude that equation (1.1) is locally solvable for $f \in G_{0}^{\frac{m}{d r}}(\Omega)$.

We observe that under assumption $i$ ) in Theorem 1.1] we obtain solvability in $C^{\infty}$, as well as $C^{\infty}$ hypoellipticity, while for the operator $\widetilde{P}$ and Theorem 1.2 we only get local solvability in $G^{\frac{m}{d r}}$.

Remark 1.3. The meaning of conditions [a] and [b] in terms of the imaginary part of the coefficients of $P$ depends on $m, d$, and the sign of $\Re b_{0}(0,0)$. Let us analyze some situations.

- If $m$ and $d$ are even and $\Re b_{0}(0,0)>0$, we can treat equation (1.1) in one of the following cases:

(a) $\Im b_{0} \equiv 0, \Im a_{l j} \leq 0(\geq 0)$ for $l$ even and $j$ odd, $\Im a_{l j} \equiv 0$ otherwise;

(b) $\Im b_{0} \equiv 0, \Im a_{l j} \leq 0(\geq 0)$ for $l$ and $j$ odd, $\Im a_{l j} \equiv 0$ otherwise.

- If $m$ is even, $d$ is odd and $\Re b_{0}(0,0)>0$, since $p(x, y, \xi, \eta)$ is quasi-elliptic for $\xi<0$, the hypotheses of Theorem [1.2 on the linear part become $\Im b_{0} \equiv 0$, $\Im a_{l j} \leq 0(\geq 0)$ for $j$ odd, $\Im a_{l j} \equiv 0$ for $j$ even.

We also observe that if $m$ and $d$ are even and $\Re b_{0}(0,0)<0$, the operator is quasielliptic and we may apply known results; cf. Mascarello-Rodino [MR and Rodino [RO].

Let us compare our result with the previously known ones. For the sake of brevity we limit our attention to a model of the form (1.2) with $d=m-1$, where 
we fix attention on the case $x=0, y=0$ :

$$
\begin{aligned}
D_{y}^{m}-D_{x}^{m-1} & +i \alpha y^{h} D_{x}^{m-1}+i\left(\beta_{1} y^{q}+\beta_{2}\right) D_{y} D_{x}^{m-2}+i \gamma D_{y}^{2} D_{x}^{m-3} \\
& +i\left(\mu_{1} y^{2 k}+\mu_{2}\right) D_{y}^{3} D_{x}^{m-4}, \quad \alpha, \beta_{1}, \beta_{2}, \gamma, \mu_{1}, \mu_{2} \in \mathbb{R}(m \geq 3) .
\end{aligned}
$$

Let us analyze first the case $\beta_{2}=\gamma=\mu_{1}=\mu_{2}=0$. For $\alpha=\beta_{1}=0$ the operator (1.9) is not hypoelliptic; observe also that for $\alpha \neq 0, \beta_{1}=0, h=1$, (1.9) is not hypoelliptic and not locally solvable, cf. Corli [C]. For $\alpha \neq 0, \beta_{1}=0, h$ even, (1.9) is hypoelliptic and locally solvable, despite the fact that $\Im b_{0}(0,0)=0$, cf. Menikoff [M], Popivanov [P1], Roberts [R]; for both $\alpha, \beta_{1} \neq 0$ and $h$ even, the operator (1.9) is not hypoelliptic if $h$ is sufficiently large with respect to $q \geq 1$, cf. Popivanov-Popov [PP], Popivanov [P2], while for $h \leq \frac{m-1}{m-2} q$ it is hypoelliptic and locally solvable, cf. Gramchev-Popivanov [GP1].

Theorem 1.1 gives new conditions on the coefficients of the terms $D_{y}^{j} D_{x}^{m-j-1}$ for models of the type (1.2), to guarantee hypoellipticity; so now we discuss the case when $\beta_{2}, \gamma, \mu_{1}, \mu_{2}$ are not all zero.

Let us observe that, if $j^{*}$ is odd, then $(i i)$, (iii) in Theorem 1.1 actually imply $\Im a_{l j}(x, y) \equiv 0$ and $\Im b_{0}(x, y) \equiv 0$ for even $j<j^{*}\left(d=m-1\right.$ implies $j+l=j^{*}+$ $\left.l^{*}=m-1\right)$; as examples of hypoelliptic and locally solvable operators characterized by Theorem 1.1 consider in this case (1.9) with $\alpha=\beta_{1}=0, \beta_{2} \neq 0\left(j^{*}=1\right)$, and (1.9) with $\alpha=\beta_{2}=\gamma=\mu_{1}=0, \beta_{1} \mu_{2}>0, q$ even $\left(j^{*}=3\right)$, having the same $b_{0}$ as the non-hypoelliptic operator $D_{y}^{m}-D_{x}^{m-1}$. If $j^{*}$ is even, then (ii) implies $\Im a_{l j} \equiv 0$ for odd $j<j^{*}$; as a corresponding example of hypoelliptic and locally solvable operator consider (1.9) with $\beta_{1}=\beta_{2}=0, \alpha \gamma>0, h$ even $\left(j^{*}=2\right)$. The order $m$ has to be chosen sufficiently large to satisfy the assumption $\frac{m-1}{2}>j^{*}$.

Now we discuss for the preceding examples the problem of local solvability in terms of Gevrey classes, arguing in the isotropic spaces $G^{\sigma}(\Omega)$. Concerning (1.9) with $h$ even, to which we may add arbitrary perturbations of lower anisotropic order, we have $\sigma$-local solvability for $\sigma<\frac{m}{m-2}$. This follows from Theorem 1.2 and is also a consequence of Marcolongo-Oliaro [MO]. The result is sharp, in the sense that when $\alpha \neq 0$ and $h=1$ (1.9) is not $\sigma$-locally solvable for $\sigma>\frac{m}{m-2}$, see Corli C. For $\alpha, \beta_{1} \neq 0, \beta_{2}=0$, it was proved by Popivanov-Popov $\mathrm{PP}$ and Popivanov [P2 that (1.9) is not $C^{\infty}$ locally solvable, and recently for $h$ even, Marcolongo [MA extended the result to $\sigma$-non-solvability for $\sigma>\frac{m}{m-2}+\varepsilon(h)$ with $\varepsilon(h) \rightarrow 0$ for $h \rightarrow \infty$. As new applications of our results in terms of $G^{\sigma}$ locally solvable operators characterized by Theorem 1.2 consider (1.9) with $\beta_{1} \neq 0, \alpha=\beta_{2}=0, q$ even $(m \geq$ 4), which is $\sigma$ locally solvable for $\sigma<\frac{m}{m-3}$, independently of lower anisotropic order perturbations. Compare in particular with $D_{y}^{m}-D_{x}^{m-1}+i y D_{y} D_{x}^{m-2}$, for which the change of sign of the imaginary part of the coefficient gives $\sigma$-non-solvability for $\sigma>\frac{m}{m-2}$. We have no change of sign and $\sigma$-solvability also in the interval $\frac{m}{m-3}>\sigma>\frac{m}{m-2}$. Moreover, let us consider (1.9) with $\beta_{1} \mu_{1}>0, \alpha=\beta_{2}=\mu_{2}=0$, $q$ even $(m \geq 10)$, which is $\sigma$ locally solvable for $\sigma<\frac{m}{m-5}$. The addition of lowerorder anisotropic terms might produce non-solvability phenomena in $C^{\infty}$ and $G^{\sigma}$ for large $\sigma$. Observe finally that if the imaginary parts of $a_{l j}$ in (1.2) vanish of high order at the origin, then the lower-order terms have no influence on the local solvability. As an example we consider the operator

$$
D_{y}^{5}-\left(1-i y^{k}\right) D_{x}^{4}+i y^{l} D_{y} D_{x}^{3}, \quad k \text { even, }
$$


which is $C^{\infty}$ locally solvable and $C^{\infty}$ hypoelliptic for $k \leq \frac{4}{3} l$; see the arguments in the book by Gramchev and Popivanov [GP1, Theorem 3.1 and Chapter 4], and see also Theorem 3.1 in Gramchev [GG]. We also observe that by applying Theorem 1.2 we obtain Gevrey $G^{\sigma}$ local solvability, $\sigma<\frac{5}{3}$, for $k>\frac{4}{3} l$, too.

\section{Gevrey-Sobolev spaces}

As a preparation for the proof of Theorem 1.2, in this section we study a class of Gevrey-Sobolev spaces defined on the strip $\mathbb{R} \times(-\delta, \delta), \delta>0$. These spaces have been introduced in the $n$-dimensional case in [MO]; here we prove some results that will be used in the next sections for the local solvability of (1.1).

Definition 2.1. We define the Gevrey-Sobolev space $\mathbb{H}_{\tau, q, r}^{s, \psi}(\mathbb{R} \times(-\delta, \delta))$ as the set of all functions $f \in L^{2}(\mathbb{R} \times(-\delta, \delta))$ such that (1.8) holds. Writing $p=\frac{1}{q}$, we shall say that $\psi(y, \xi)$ is a weight function of order $(r, p)$. The operator $e^{\tau \psi\left(y, D_{x}\right)}$ acts on the function $f$ in the following way:

$$
e^{\tau \psi\left(y, D_{x}\right)} f(x, y)=\frac{1}{2 \pi} \int e^{i x \xi} e^{\tau \psi(y, \xi)} \widetilde{f}(y, \xi) d \xi
$$

where $\tilde{f}(y, \xi)=\int e^{-i x \xi} f(x, y) d x$, and $H_{p}^{s}(\mathbb{R} \times(-\delta, \delta))$, for $s$ integer, is the space of all $g(x, y) \in L^{2}(\mathbb{R} \times(-\delta, \delta))$ such that

$$
\|f(x, y)\|_{H_{p}^{s}}^{2}:=\sum_{k=0}^{s} \int\left(1+|\xi|^{2 p}\right)^{s-k}\left|D_{y}^{k} \tilde{f}(y, \xi)\right|^{2} d \xi d y<+\infty
$$

the definition of $H_{p}^{s}(\mathbb{R} \times(-\delta, \delta))$ extends to every $s>0$ by interpolation.

Remark 2.1. The operator $e^{\tau \psi\left(y, D_{x}\right)}$ and its inverse $e^{-\tau \psi\left(y, D_{x}\right)}$ establish an isometry between the Hilbert spaces $\mathbb{H}_{\tau, q, r}^{s, \psi}(\mathbb{R} \times(-\delta, \delta))$ and $H_{p}^{s}(\mathbb{R} \times(-\delta, \delta))$.

We need to introduce suitable equivalent norms on the spaces $\mathbb{H}_{\tau, q, r}^{s, \psi}(\mathbb{R} \times(-\delta, \delta))$.

First we recall that the following identities hold:

$$
\begin{gathered}
D_{y}^{j} e^{\tau \psi\left(y, D_{x}\right)}=\sum_{h=0}^{j} q_{j-h}^{(j)}\left(y, D_{x}\right) e^{\tau \psi\left(y, D_{x}\right)} D_{y}^{h}, \\
e^{\tau \psi\left(y, D_{x}\right)} D_{y}^{j}=\sum_{h^{\prime}=0}^{j} r_{j-h^{\prime}}^{(j)}\left(y, D_{x}\right) D_{y}^{h^{\prime}} e^{\tau \psi\left(y, D_{x}\right)}, \\
D_{x}^{k} e^{\tau \psi\left(y, D_{x}\right)}=e^{\tau \psi\left(y, D_{x}\right)} D_{x}^{k}
\end{gathered}
$$

for every $k, j \in \mathbb{Z}_{+}$, where the symbols of the pseudo-differential operators $q_{j-l}^{(j)}\left(y, D_{x}\right)$ and $r_{j-l}^{(j)}\left(y, D_{x}\right)$ belong to the Hörmander class $S_{1,0}^{p r(j-l)}((-\delta, \delta) \times \mathbb{R})$. In particular, we obtain that

$$
q_{0}^{(j)}\left(y, D_{x}\right)=1, \quad q_{1}^{(j)}\left(y, D_{x}\right)=j \tau\left(D_{y} \psi\right)\left(y, D_{x}\right)
$$

and

$$
r_{0}^{(j)}\left(y, D_{x}\right)=1, \quad r_{1}^{(j)}\left(y, D_{x}\right)=-j \tau\left(D_{y} \psi\right)\left(y, D_{x}\right)
$$

For details, see [MO Lemma 1.1], in which (2.2)-(2.4) are proved in the $n$-dimensional case. 
Definition 2.2. We say that a function $f$ belongs to the space $H_{\left(p_{1}, p_{2}\right)}^{s}\left(\mathbb{R}^{2}\right), s \geq 0$, $0<p_{1} \leq 1,0<p_{2} \leq 1$, if and only if $f \in L^{2}\left(\mathbb{R}^{2}\right)$ and

$$
\|f(x, y)\|_{H_{\left(p_{1}, p_{2}\right)}^{s}\left(\mathbb{R}^{2}\right)}^{2}:=\int\left(1+|\xi|^{2 p_{1}}+|\eta|^{2 p_{2}}\right)^{s}|\widehat{f}(\xi, \eta)|^{2} d \xi d \eta<\infty .
$$

Lemma 2.1. Let $f \in H_{\left(p_{1}, p_{2}\right)}^{s}\left(\mathbb{R}^{2}\right), s \geq 0$; let $t$ and $h$ be such that $\frac{t}{p_{1}}+\frac{h}{p_{2}} \leq s$. Then

$$
\left\|\left\langle D_{x}\right\rangle^{t}\left\langle D_{y}\right\rangle^{h} f\right\|_{L^{2}\left(\mathbb{R}^{2}\right)} \leq\left\|\left\langle D_{x}\right\rangle^{p_{1} s} f\right\|_{L^{2}\left(\mathbb{R}^{2}\right)}+\left\|\left\langle D_{y}\right\rangle^{p_{2} s} f\right\|_{L^{2}\left(\mathbb{R}^{2}\right)}+\|f\|_{L^{2}\left(\mathbb{R}^{2}\right)},
$$

where $\left\langle D_{x}\right\rangle^{l}$ and $\left\langle D_{y}\right\rangle^{l^{\prime}}$ are the pseudo-differential operators with symbols $|\xi|^{l}$ and $|\eta|^{l^{\prime}}$, respectively; we write $(\xi, \eta)$ for the dual variables of $(x, y)$.

Proof. Let $K=[-1,1] \times[-1,1]$; we obtain

$$
\begin{aligned}
\left\|\left\langle D_{x}\right\rangle^{t}\left\langle D_{y}\right\rangle^{h} f\right\|_{L^{2}\left(\mathbb{R}^{2}\right)}^{2} & \leq \int_{\mathbb{R}^{2} \backslash K}\left(|\xi|^{2 p_{1} s}+|\eta|^{2 p_{2} s}\right)|\widehat{f}(\xi, \eta)|^{2} d \xi d \eta+\int_{K}|\widehat{f}(\xi, \eta)|^{2} d \xi d \eta \\
& \leq\left\|\left\langle D_{x}\right\rangle^{p_{1} s} f\right\|_{L^{2}\left(\mathbb{R}^{2}\right)}^{2}+\left\|\left\langle D_{y}\right\rangle^{p_{2} s} f\right\|_{L^{2}\left(\mathbb{R}^{2}\right)}^{2}+\|f\|_{L^{2}\left(\mathbb{R}^{2}\right)}^{2} .
\end{aligned}
$$

Remark 2.2. Let us suppose that $p_{1}, p_{2} \in \mathbb{Q}$, and let $s$ be a positive integer such that $p_{j} s$ is an integer, $j=1,2$. By Lemma 2.1 we have that an equivalent norm in $H_{\left(p_{1}, p_{2}\right)}^{s}\left(\mathbb{R}^{2}\right)$ is given by the following expression:

$$
\left\|D_{x}^{p_{1} s} f\right\|_{L^{2}\left(\mathbb{R}^{2}\right)}+\left\|D_{y}^{p_{2} s} f\right\|_{L^{2}\left(\mathbb{R}^{2}\right)}+\|f\|_{L^{2}\left(\mathbb{R}^{2}\right)} .
$$

Lemma 2.2. Consider $u \in H_{p}^{s}(\mathbb{R} \times(-\delta, \delta))$ with $p$ a rational number and $s$ a positive integer such that ps is an integer; we write $\Theta$ for $\mathbb{R} \times(-\delta, \delta)$. Then there exists a function $l_{s, \Theta} u \in H_{(p, 1)}^{s}\left(\mathbb{R}^{2}\right)$ that extends $u$. Moreover, we can find a constant $C$ such that:

$$
\begin{aligned}
\left\|l_{s, \Theta} u\right\|_{L^{2}\left(\mathbb{R}^{2}\right)} & \leq C\|u\|_{L^{2}(\mathbb{R} \times(-\delta, \delta))}, \\
\left\|D_{x}^{p s} l_{s, \Theta} u\right\|_{L^{2}\left(\mathbb{R}^{2}\right)} & \leq C\left\|D_{x}^{p s} u\right\|_{L^{2}(\mathbb{R} \times(-\delta, \delta))}, \\
\left\|D_{y}^{s} l_{s, \Theta} u\right\|_{L^{2}\left(\mathbb{R}^{2}\right)} & \leq C\left\|D_{y}^{s} u\right\|_{L^{2}(\mathbb{R} \times(-\delta, \delta))}
\end{aligned}
$$

This lemma can be proved using an argument similar to the one developed in the isotropic case by Egorov and Schulze in [ES, Theorem 27]. The proof is omitted.

Remark 2.3. Observe that $\|u\|_{L^{2}(\mathbb{R} \times(-\delta, \delta))} \leq\left\|l_{s, \Theta} u\right\|_{L^{2}\left(\mathbb{R}^{2}\right)}, l_{s, \Theta} u$ being an extension of $u$; so $\|u\|_{L^{2}(\mathbb{R} \times(-\delta, \delta))}$ and $\left\|l_{s, \Theta} u\right\|_{L^{2}\left(\mathbb{R}^{2}\right)}$ are equivalent. In the same way we obtain that $\left\|D_{x}^{p s} u\right\|_{L^{2}(\mathbb{R} \times(-\delta, \delta))}$ and $\left\|D_{y}^{s} u\right\|_{L^{2}(\mathbb{R} \times(-\delta, \delta))}$ are equivalent to $\left\|D_{x}^{p s} l_{s, \Theta} u\right\|_{L^{2}\left(\mathbb{R}^{2}\right)}$ and $\left\|D_{y}^{s} l_{s, \Theta} u\right\|_{L^{2}\left(\mathbb{R}^{2}\right)}$ respectively. So if $s$ is an integer such that $p s$ is an integer and $f \in H_{p}^{s}(\mathbb{R} \times(-\delta, \delta))$, using Lemma 2.2 and Remark 2.2 we can prove that an equivalent norm in the space $H_{p}^{s}(\mathbb{R} \times(-\delta, \delta))$ is given by the following expression:

$$
\left\|D_{x}^{p s} f\right\|_{L^{2}(\mathbb{R} \times(-\delta, \delta))}+\left\|D_{y}^{s} f\right\|_{L^{2}(\mathbb{R} \times(-\delta, \delta))}+\|f\|_{L^{2}(\mathbb{R} \times(-\delta, \delta))} .
$$

Theorem 2.1. Let us fix $s>0, \tau>0, r \in(0,1], p \in(0,1]$ and assume that $s$ is a positive integer such that $p s$ is an integer. Then the following norms are equivalent:

(a) $\|f\|_{\mathbb{H}_{T, q, r}^{s, r}(\mathbb{R} \times(-\delta, \delta))}$; 
(b) $\sum_{\frac{l}{p}+j \leq s}\left\|D_{x}^{l} D_{y}^{j} e^{\tau \psi\left(y, D_{x}\right)} f\right\|_{L^{2}(\mathbb{R} \times(-\delta, \delta))}$;

(c) $\sum_{\frac{l}{p}+j \leq s}\left\|e^{\tau \psi\left(y, D_{x}\right)} D_{x}^{l} D_{y}^{j} f\right\|_{L^{2}(\mathbb{R} \times(-\delta, \delta))}$;

(d) $\sum_{\frac{l_{1}+l_{2}}{p}+j_{1}+j_{2} \leq s}\left\|D_{x}^{l_{1}} D_{y}^{j_{1}} e^{\tau \psi\left(y, D_{x}\right)} D_{x}^{l_{2}} D_{y}^{j_{2}} f\right\|_{L^{2}(\mathbb{R} \times(-\delta, \delta))}$.

Proof. (a) $\Leftrightarrow$ (b). Using Lemma 2.1 and Lemma 2.2 we obtain

$$
\begin{aligned}
\sum_{\frac{l}{p}+j \leq s} & \left\|D_{x}^{l} D_{y}^{j} e^{\tau \psi\left(y, D_{x}\right)} f\right\|_{L^{2}(\mathbb{R} \times(-\delta, \delta))} \\
\leq & \sum_{\frac{l}{p}+j \leq s}\left\|D_{x}^{l} D_{y}^{j} l_{s, \Theta} e^{\tau \psi\left(y, D_{x}\right)} f\right\|_{L^{2}\left(\mathbb{R}^{2}\right)} \\
\leq & C\left(\left\|D_{x}^{p s} l_{s, \Theta} e^{\tau \psi\left(y, D_{x}\right)} f\right\|_{L^{2}\left(\mathbb{R}^{2}\right)}+\left\|D_{y}^{s} l_{s, \Theta} e^{\tau \psi\left(y, D_{x}\right)} f\right\|_{L^{2}\left(\mathbb{R}^{2}\right)}\right. \\
& \left.\quad+\left\|l_{s, \Theta} e^{\tau \psi\left(y, D_{x}\right)} f\right\|_{L^{2}\left(\mathbb{R}^{2}\right)}\right) \\
\leq & C_{1}\left(\left\|D_{x}^{p s} e^{\tau \psi\left(y, D_{x}\right)} f\right\|_{L^{2}(\mathbb{R} \times(-\delta, \delta))}+\left\|D_{y}^{s} e^{\tau \psi\left(y, D_{x}\right)} f\right\|_{L^{2}(\mathbb{R} \times(-\delta, \delta))}\right. \\
& \left.\quad+\left\|e^{\tau \psi\left(y, D_{x}\right)} f\right\|_{L^{2}(\mathbb{R} \times(-\delta, \delta))}\right) \\
\leq & \|f\|_{\mathbb{H}_{\tau, q, r}^{s, \psi}(\mathbb{R} \times(-\delta, \delta))}
\end{aligned}
$$

On the other hand, since $\|f\|_{\mathbb{H}_{T, q, r}^{s, \psi}(\mathbb{R} \times(-\delta, \delta))} \leq\left\|l_{s, \Theta}\left(e^{\tau \psi\left(y, D_{x}\right)} f\right)\right\|_{H_{(p, 1)}^{s}\left(\mathbb{R}^{2}\right)}$, by Remark 2.2 and Lemma 2.2 we have

$$
\|f\|_{\mathbb{H}_{T, q, r}^{s, \psi}(\mathbb{R} \times(-\delta, \delta))} \leq C \sum_{\frac{l}{p}+j \leq s}\left\|D_{x}^{l} D_{y}^{j} e^{\tau \psi\left(y, D_{x}\right)} f\right\|_{L^{2}(\mathbb{R} \times(-\delta, \delta))} .
$$

(b) $\Leftrightarrow$ (c). Using Lemma 2.1, Lemma 2.2, Remark 2.3 and the identities (2.2)(2.4), we obtain

$$
\begin{aligned}
& \sum_{\frac{l}{p}+j \leq s}\left\|e^{\tau \psi\left(y, D_{x}\right)} D_{x}^{l} D_{y}^{j} f\right\|_{L^{2}(\mathbb{R} \times(-\delta, \delta))} \\
& \leq C \sum_{\frac{l}{p}+j \leq s} \sum_{h=0}^{j}\left\|r_{j-h}^{(j)}\left(y, D_{x}\right) D_{y}^{h} D_{x}^{l} e^{\tau \psi\left(y, D_{x}\right)} f\right\|_{L^{2}(\mathbb{R} \times(-\delta, \delta))} \\
& \leq C_{1} \sum_{\frac{l}{p}+j \leq s} \sum_{h=0}^{j} \sum_{k=0}^{j-h}\left\|\left\langle D_{x}\right\rangle^{r p k+l} D_{y}^{h} l_{s, \Theta} e^{\tau \psi\left(y, D_{x}\right)} f\right\|_{L^{2}\left(\mathbb{R}^{2}\right)} \\
& \leq C_{2}\left(\left\|D_{x}^{p s} l_{s, \Theta} e^{\tau \psi\left(y, D_{x}\right)} f\right\|_{L^{2}\left(\mathbb{R}^{2}\right)}+\left\|D_{y}^{s} l_{s, \Theta} e^{\tau \psi\left(y, D_{x}\right)} f\right\|_{L^{2}\left(\mathbb{R}^{2}\right)}\right. \\
&\left.\quad+\left\|l_{s, \Theta} e^{\tau \psi\left(y, D_{x}\right)} f\right\|_{L^{2}\left(\mathbb{R}^{2}\right)}\right) \\
& \leq C_{2} \sum_{\frac{l}{p}+j \leq s}\left\|D_{x}^{l} D_{y}^{j} e^{\tau \psi\left(y, D_{x}\right)} f\right\|_{L^{2}(\mathbb{R} \times(-\delta, \delta))} .
\end{aligned}
$$

In the opposite direction we may use similar arguments. By the same arguments we have (c) $\Leftrightarrow(d)$.

Now we prove some important results that will be used in Section 3 for the solvability of the semilinear equation. 
Theorem 2.2. Let $0<p<1$ and $0<r<1$. Let $f \in G_{0}^{\left(\bar{q}, q_{2}\right)}$ with $\bar{q}=\frac{1}{r p}$ and $q_{2}>1$. Then for all $s>0$ and for every weight function $\psi(y, \xi)$ of order $(r, p)$ there exists $\tau>0$ such that $f \in \mathbb{H}_{\tau, q, r}^{s, \psi}$, where $q=\frac{1}{p}$.

Proof. First we observe that $\mathbb{H}_{\tau, q, r}^{s, \psi} \subset \mathbb{H}_{\tau, q, r}^{t, \psi}$ for $s>t$; then, without loss of generality, we may assume that $s$ is a positive integer such that $p s$ is an integer. By Theorem 2.1 we can write

$$
\|f\|_{\mathbb{H}_{T, q, r}^{s, \psi}}=\sum_{\frac{\alpha_{1}}{p}+\alpha_{2} \leq s}\left\|e^{\tau \psi(y, \xi)} \widetilde{D^{\alpha} f}(y, \xi)\right\|_{L^{2}} .
$$

The functions $D_{(x, y)}^{\alpha} f(x, y)$ obviously belong to $G_{0}^{\left(\bar{q}, q_{2}\right)}(\mathbb{R} \times(-\delta, \delta))$ for every multiindex $\alpha=\left(\alpha_{1}, \alpha_{2}\right)$ such that $\frac{\alpha_{1}}{p}+\alpha_{2} \leq s$, with the same constant $C_{K}$ as in Definition 1.1, depending on $s$. For every integer $k$, applying the Fourier transform with respect to $x$ we can find $b \in \mathbb{R}$, depending on $\operatorname{supp} f$, such that

$$
|\xi|^{k}\left|\widetilde{D^{\alpha} f}(y, \xi)\right| \leq \int_{-b}^{b} \bar{C}^{k+1}(k !)^{\bar{q}} d x \leq C\left(C^{k}(k !)^{\bar{q}}\right) .
$$

It follows immediately that $(1+|\xi|)^{k}\left|\widetilde{D^{\alpha} f}(y, \xi)\right| \leq C\left(C_{1}^{k}(k !)^{\bar{q}}\right)$ for all integers $k$, where we suppose $C \geq 1$. So we have

$$
\sum_{k} \frac{1}{k !}\left(\frac{1+|\xi|}{2 C_{1}}\right)^{p k r}\left|\widetilde{D^{\alpha} f}(y, \xi)\right|^{r p} \leq C^{p r} \sum_{k}\left(\frac{1}{2^{r p}}\right)^{k}
$$

therefore we obtain

$$
\left|\widetilde{D^{\alpha} f}(y, \xi)\right| \leq K e^{-M(1+|\xi|)^{r p}} .
$$

Since $\psi(y, \xi)$ is a weight function of order $(r, p)$, we can find a constant $C$ such that

$$
\left|e^{\tau \psi(y, \xi)} \widetilde{D^{\alpha} f}(y, \xi)\right| \leq K e^{(\tau C-M)(1+|\xi|)^{r p}} .
$$

Choosing $\tau<\frac{M}{2 C}$, we can conclude that $e^{\tau \psi(y, \xi)} \widetilde{D^{\alpha} f}(y, \xi) \in L^{2}$ for $\frac{\alpha_{1}}{p}+\alpha_{2} \leq s$; using (2.9), we have that $f \in \mathbb{H}_{\tau, q, r}^{s, \psi}$.

Remark 2.4. Let $0<p<1$ and $0<r<1$. Let $f \in G_{0}^{\left(q_{1}, q_{2}\right)}(\mathbb{R} \times(-\delta, \delta))$ with $1<q_{1}<\frac{1}{r p}$ and $q_{2}>1$. Then, for every $s>0$, for every weight function $\psi(y, \xi)$ of order $(r, p)$ and for every $\tau>0$, we have that $f \in \mathbb{H}_{\tau, q, r}^{s, \psi}(\mathbb{R} \times(-\delta, \delta))$, where $q=\frac{1}{p}$.

Theorem 2.3. Let $\psi(y, \xi)$ be essentially subadditive with respect to $\xi$, i.e.,

$$
\psi\left(y, \xi_{1}+\xi_{2}\right) \leq \psi\left(y, \xi_{1}\right)+\psi\left(y, \xi_{2}\right)+C,
$$

cf. GR. Let $s_{0} \geq \frac{p+1}{2 p}$ satisfy the assumptions of Theorem 2.1. Then for every $s>s_{0}$ the space $\mathbb{H}_{\tau, q, r}^{s, \psi}(\mathbb{R} \times(-\delta, \delta))$ is an algebra, and there exists a constant $C_{s}$ such that

$$
\|u v\|_{\mathbb{H}_{T, q, r}^{s, \psi}} \leq C_{s}\|u\|_{\mathbb{H}_{T, q, r}^{s, \psi}}\|v\|_{\mathbb{H}_{T, q, r}^{s, \psi}} .
$$

Proof. We begin by proving Theorem 2.3 with $s$ a positive integer such that $p s$ is an integer. Using Theorem 2.1, (c) and applying the Leibniz rule, we obtain

$$
\|u v\|_{\mathbb{H}_{T, q, r}^{s, \psi}} \leq C \sum_{\frac{\beta_{1}+\gamma_{1}}{p}+\beta_{2}+\gamma_{2} \leq s}\left\|e^{\tau \psi\left(y, D_{x}\right)}\left(e^{-\tau \psi\left(y, D_{x}\right)} u_{\beta} e^{-\tau \psi\left(y, D_{x}\right)} v_{\gamma}\right)\right\|_{L^{2}}
$$


where $u_{\beta}(x, y)=e^{\tau \psi\left(y, D_{x}\right)} D^{\beta} u, v_{\gamma}(x, y)=e^{\tau \psi\left(y, D_{x}\right)} D^{\gamma} v$ and the norms are in $\mathbb{R} \times(-\delta, \delta)$. Applying the Fourier transform with respect to $x$ and writing $*_{(\xi)}$ for the convolution in the $\xi$ variable, we have

$$
\|u v\|_{\mathbb{H}_{\tau, q, r}^{s, \psi}} \leq C_{1} \sum_{\frac{\beta_{1}+\gamma_{1}}{p}+\beta_{2}+\gamma_{2} \leq s}\left\|e^{\tau \psi(y, \xi)}\left(\left(e^{-\tau \psi(y, \xi)} \widetilde{u}_{\beta}\right) *(\xi)\left(e^{-\tau \psi(y, \xi)} \widetilde{v}_{\gamma}\right)\right)(y, \xi)\right\|_{L^{2}},
$$

where $\widetilde{u}_{\beta}$ and $\widetilde{v}_{\gamma}$ stand for the partial Fourier transform with respect to $x$ of $u_{\beta}$ and $v_{\gamma}$ respectively. The function $\psi(y, \xi)$ being essentially subadditive with respect to $\xi$, it follows immediately that $e^{\tau \psi(y, \xi)-\tau \psi(y, \xi-\mu)-\tau \psi(y, \mu)} \leq e^{C}$, and so

$$
\|u v\|_{\mathbb{H}_{T, \psi}^{s, \psi}, r} \leq C_{2} \sum_{\frac{\beta_{1}+\gamma_{1}}{p}+\beta_{2}+\gamma_{2} \leq s}\left\|u_{\beta} v_{\gamma}\right\|_{L^{2}}
$$

Since we have required $s>\frac{p+1}{2 p}$, at least one of the inequalities $\frac{\beta_{1}}{p}+\beta_{2}<s-\frac{p+1}{4 p}$ and $\frac{\gamma_{1}}{p}+\gamma_{2}<s-\frac{p+1}{4 p}$ must be satisfied. Let $\rho>0$ be such that

$$
\begin{aligned}
& \left\{\left(\alpha_{1}, \alpha_{2}\right) \in \mathbb{Z}_{+}^{2}: \frac{\alpha_{1}}{p}+\alpha_{2}<s-\frac{p+1}{4 p}\right\} \\
& =\left\{\left(\alpha_{1}, \alpha_{2}\right) \in \mathbb{Z}_{+}^{2}: \frac{\alpha_{1}}{p}+\alpha_{2} \leq s-\frac{p+1}{4 p}-\rho\right\} .
\end{aligned}
$$

Using Lemma 2.2 and Young's estimates, we have

$$
\begin{aligned}
\|u v\|_{\mathbb{H}_{T, q, r}^{s, \psi}} \leq & C_{3} \sum_{\substack{\frac{\beta_{1}}{p}+\beta_{2} \leq s-\frac{p+1}{4 p}-\rho \\
\frac{\gamma_{1}}{p}+\gamma_{2} \leq s}}\left\|\left(l_{\frac{p+1}{4 p}+\rho, \Theta} u_{\beta}\right)^{\wedge}\right\|_{L^{1}\left(\mathbb{R}^{2}\right)}\left\|\left(l_{0, \Theta} v_{\gamma}\right)^{\wedge}\right\|_{L^{2}\left(\mathbb{R}^{2}\right)} \\
& +C_{3} \sum_{\substack{\frac{\beta_{1}}{p}+\beta_{2} \leq s \\
\frac{\gamma_{1}}{p}+\gamma_{2} \leq s-\frac{p+1}{4 p}-\rho}}\left\|\left(l_{0, \Theta} u_{\beta}\right)^{\wedge}\right\|_{L^{2}\left(\mathbb{R}^{2}\right)}\left\|\left(l_{\frac{p+1}{4 p}+\rho, \Theta} v_{\gamma}\right)^{\wedge}\right\|_{L^{1}\left(\mathbb{R}^{2}\right)} .
\end{aligned}
$$

Since $u, v \in \mathbb{H}_{\tau, q, r}^{s, \psi}(\mathbb{R} \times(-\delta, \delta))$ and $\frac{\beta_{1}}{p}+\beta_{2} \leq s, \frac{\gamma_{1}}{p}+\gamma_{2} \leq s$, Theorem 2.1 and (2.7) assure us that $\left\|\left(l_{0, \Theta} u_{\beta}\right)^{\wedge}\right\|_{L^{2}\left(\mathbb{R}^{2}\right)} \leq\|u\|_{\mathbb{H}_{\tau, q, r}^{s, \psi}}$ and $\left\|\left(l_{0, \Theta} v_{\gamma}\right)^{\wedge}\right\|_{L^{2}\left(\mathbb{R}^{2}\right)} \leq\|v\|_{\mathbb{H}_{\tau, q, r}^{s, \psi}}$. Moreover, if $\frac{\beta_{1}}{p}+\beta_{2} \leq s-\frac{p+1}{4 p}-\rho$, by the Hölder inequality and (2.7) we have

$$
\begin{aligned}
& \left\|\left(l_{\frac{p+1}{4 p}+\rho, \Theta} u_{\beta}\right)^{\wedge}(\xi, \eta)\right\|_{L^{1}\left(\mathbb{R}^{2}\right)} \\
& \quad \leq C_{4}\left\|\left(1+|\xi|^{p\left(\frac{p+1}{4 p}+\rho\right)}+|\eta|^{\frac{p+1}{4 p}+\rho}\right)\left(l_{\frac{p+1}{4 p}+\rho, \Theta} u_{\beta}\right)^{\wedge}\right\|_{L^{2}\left(\mathbb{R}^{2}\right)} \leq C_{5}\|u\|_{\mathbb{H}_{T, q, r}^{s, \psi}} .
\end{aligned}
$$

The same arguments allow us to show that $\left\|\left(l_{\frac{p+1}{4 p}+\rho, \Theta} v_{\gamma}\right)^{\wedge}\right\|_{L^{1}} \leq C\|v\|_{\mathbb{H}_{\tau, q, r}^{s, \psi}}$. So (2.10) holds. By interpolation, the result remains valid for every $s>s_{0}$.

\section{ANAlysis of the Linear EQUATION AND PROOF OF THEOREMS 1.1] AND 1.2}

Let us consider the operator (1.2), where we take the coefficients $b_{0}(x, y)$ and $a_{l j}(x, y)$ in the space $G^{\left(q_{1}, q_{2}\right)}(\Omega)$ with $1<q_{1}<\frac{m}{d r}, q_{2}>1$. We choose $t$ in (1.2) in such a way that there exist two integers $\bar{l}$ and $\bar{j}$ for which $\bar{l} \frac{m}{d}+\bar{j}=m-t$.

Let us observe that the operator $P\left(x, y, D_{x}, D_{y}\right)$ does not involve the terms $a_{l j}(x, y) D_{x}^{l} D_{y}^{j}$ with order $l \frac{m}{d}+j<m-t$; these terms have order $l \frac{m}{d}+j \leq m-t-\epsilon_{t}$, 
where $\epsilon_{t}$ is given by the following expression:

$$
\epsilon_{t}= \begin{cases}\min _{h \in I_{t}}\left(\mathcal{M}\left(\frac{d}{m}(m-t-h)\right) \frac{m}{d}\right), & I_{t} \neq \emptyset, \\ 1, & I_{t}=\emptyset .\end{cases}
$$

The symbol $\mathcal{M}(b)$ stands for the decimal part of $b$, and $I_{t}=\{h \in[0, m-t), h \in$ $\left.\mathbb{N}: \frac{d}{m}(m-t-h) \notin \mathbb{N}\right\}$. We deal with the local solvability at the origin of the equation

$$
P\left(x, y, D_{x}, D_{y}\right) v(x, y)=f(x, y),
$$

$P$ as in (1.2); so it is not restrictive to multiply the coefficients $b_{0}(x, y)$ and $a_{l j}(x, y)$ by a function $\chi(x, y) \in G_{0}^{\left(q_{1}, q_{2}\right)}(\Omega)$ with support in a neighborhood of the origin. Thus, we can suppose that $b_{0}(x, y)$ and $a_{l j}(x, y)$ are compactly supported.

Now we fix a weight function $\psi(y, \xi)$ of order $\left(r, \frac{d}{m}\right)$, essentially subadditive with respect to $\xi$; for every $s \geq s_{0}$ and $\tau$ we consider the anisotropic Gevrey-Sobolev space $\mathbb{H}_{\tau, \frac{m}{d}, r}^{s, \psi}(\mathbb{R} \times(-\delta, \delta))$.

For an arbitrary real number $q \geq 1$ we set:

(i) $\mathbb{H}_{\tau, q, r, \text { comp }}^{s, \psi}(\Omega):=\left\{f \in \mathbb{H}_{\tau, q, r}^{s, \psi}(\mathbb{R} \times(-\delta, \delta))\right.$ with compact support contained in $\Omega\}$;

(ii) $\mathbb{H}_{\tau, q, r, l o c}^{s, \psi}(\Omega):=\left\{f \in \mathcal{D}^{\prime}(\Omega)\right.$ : for every $\varphi \in G_{0}^{\left(q_{1}, q_{2}\right)}(\Omega), 1<q_{1}<\frac{q}{r}, q_{2}>1$, we have $\left.\varphi f \in \mathbb{H}_{\tau, q, r, c o m p}^{s, \psi}(\Omega)\right\}$;

(iii) $\mathbb{H}_{\tau, q, r}^{s, \psi}(\Omega):=\{f$ such that $f$ is a restriction to $\Omega$ of a function belonging to $\left.\mathbb{H}_{\tau, q, r}^{s, \psi}(\mathbb{R} \times(-\delta, \delta))\right\}$.

It follows from Theorem 2.1 Theorem 2.3 and Remark 2.4 that, for $s \geq s_{0}$,

$$
P\left(x, y, D_{x}, D_{y}\right): \mathbb{H}_{\tau, \frac{m}{d}, r}^{s, \psi}(\mathbb{R} \times(-\delta, \delta)) \longrightarrow \mathbb{H}_{\tau, \frac{m}{d}, r}^{s-m, \psi}(\mathbb{R} \times(-\delta, \delta)) .
$$

Moreover, the operator $P\left(x, y, D_{x}, D_{y}\right)$ can be regarded as a continuous map

$$
P\left(x, y, D_{x}, D_{y}\right): \mathbb{H}_{\tau, \frac{m}{d}, r, c o m p}^{s, \psi}(\Omega) \longrightarrow \mathbb{H}_{\tau, \frac{m}{d}, r, l o c}^{s-m, \psi}(\Omega),
$$

or also as a continuous map

$$
P\left(x, y, D_{x}, D_{y}\right): \mathbb{H}_{\tau, \frac{m}{d}, r}^{s, \psi}(\Omega) \longrightarrow \mathbb{H}_{\tau, \frac{m}{d}, r}^{s-m, \psi}(\Omega) .
$$

Now let us consider the following operator:

$$
\widetilde{P}\left(x, y, D_{x}, D_{y}\right):=e^{\tau \psi\left(y, D_{x}\right)} P\left(x, y, D_{x}, D_{y}\right) e^{-\tau \psi\left(y, D_{x}\right)} .
$$

By Remark 2.1 and the previous considerations we have

$$
P\left(x, y, D_{x}, D_{y}\right)=e^{-\tau \psi\left(y, D_{x}\right)} \widetilde{P}\left(x, y, D_{x}, D_{y}\right) e^{\tau \psi\left(y, D_{x}\right)},
$$

and, moreover,

$$
\widetilde{P}\left(x, y, D_{x}, D_{y}\right): H_{\frac{d}{m}}^{s}(\mathbb{R} \times(-\delta, \delta)) \longrightarrow H_{\frac{d}{m}}^{s-m}(\mathbb{R} \times(-\delta, \delta)) .
$$

Now we want to analyze the conjugate operator $\widetilde{P}\left(x, y, D_{x}, D_{y}\right)$; to this aim we start from the following proposition. 
Proposition 3.1. Let $0<p \leq 1$ and $0<r<1$; as usual we set $q=\frac{1}{p}$. Let us fix a function $a(x, y) \in G_{0}^{\left(q_{1}, q_{2}\right)}, 1<q_{1}<\frac{q}{r}, q_{2}>1$, and a weight function $\psi(y, \xi)$ of order $(r, p)$, essentially subadditive with respect to $\xi$. Then

$$
\begin{aligned}
e^{\tau \psi\left(y, D_{x}\right)} a(x, y) e^{-\tau \psi\left(y, D_{x}\right)} & \\
& =a(x, y)+Q_{-(q-r)}\left(x, y, D_{x}\right)+Q_{-2(q-r)}\left(x, y, D_{x}\right),
\end{aligned}
$$

where the symbols $q_{-j(q-r)}(x, y, \xi)$ of the operators $Q_{-j(q-r)}\left(x, y, D_{x}\right), j=1,2$, satisfy the following conditions:

$$
\begin{gathered}
q_{-(q-r)}(x, y, \xi)=\tau \partial_{\xi} \psi(y, \xi)\left(D_{x} a\right)(x, y) \in S_{1,0}^{-(q-r) p}(\Omega \times \mathbb{R}) ; \\
q_{-2(q-r)}(x, y, \xi) \in S_{1,0}^{-2(q-r) p}(\Omega \times \mathbb{R}) .
\end{gathered}
$$

Proof. Setting $Q_{a}\left(x, y, D_{x}\right)=e^{\tau \psi\left(y, D_{x}\right)} a(x, y) e^{-\tau \psi\left(y, D_{x}\right)}$ and using the standard properties of the oscillatory integrals, we easily obtain that the symbol $q_{a}(x, y, \xi)$ of the operator $Q_{a}\left(x, y, D_{x}\right)$ is given by

$$
q_{a}(x, y, \xi)=\int e^{i x \eta} e^{\tau \psi(y, \xi+\eta)-\tau \psi(y, \xi)} \widetilde{a}(\eta, y) d \eta,
$$

where we write $d \eta=(2 \pi)^{-1} d \eta$ and we denote as usual by $\widetilde{a}(\eta, y)$ the Fourier transform of $a(x, y)$ with respect to the $x$ variable. Applying the Taylor formula to $e^{\tau \psi(y, \xi+\eta)}$, we obtain

$$
\begin{aligned}
& e^{\tau \psi(y, \xi+\eta)-\tau \psi(y, \xi)} \\
& \quad=1+\tau \partial_{\xi} \psi(y, \xi) \eta+\sum_{n=2}^{N-1} \frac{1}{n !}\left(e^{-\tau \psi(y, \xi)} \partial_{\xi}^{n} e^{\tau \psi(y, \xi)}\right) \eta^{n}+r_{N}(y, \xi, \eta),
\end{aligned}
$$

where

$$
\begin{aligned}
& r_{N}(y, \xi, \eta) \\
& =\frac{\eta^{N}}{(N-1) !} \int_{0}^{1} e^{-\tau \psi(y, \xi+t \eta)} \partial_{\xi}^{N}\left(e^{\tau \psi(y, \xi+t \eta)}\right) e^{\tau \psi(y, \xi+t \eta)-\tau \psi(y, \xi)}(1-t)^{N-1} d t .
\end{aligned}
$$

From (3.8) and the standard properties of the Fourier transform it follows immediately that

$$
\begin{aligned}
q_{a}(x, y, \xi)= & a(x, y)+\tau \partial_{\xi} \psi(y, \xi)\left(D_{x} a\right)(x, y) \\
& \quad+\sum_{n=2}^{N-1} \frac{1}{n !} \int e^{i x \eta} e^{-\tau \psi(y, \xi)} \partial_{\xi}^{n} e^{\tau \psi(y, \xi)} \widetilde{D_{x}^{n} a}(y, \eta) d \eta \\
& \quad+\int e^{i x \eta} r_{N}(y, \xi, \eta) \widetilde{a}(y, \eta) d \eta \\
= & a(x, y)+q_{-(q-r)}(x, y, \xi)+\left(q_{-2(q-r)}^{(1)}(x, y, \xi)+q_{-2(q-r)}^{(2)}(x, y, \xi)\right) .
\end{aligned}
$$

Using the Leibniz rule, Definition 1.1 and the fact that $\psi \in S_{1,0}^{r p}((-\delta, \delta) \times \mathbb{R})$, cf. Definition 2.1 we easily obtain that $q_{-(q-r)} \in S_{1,0}^{-(q-r) p}(\Omega \times \mathbb{R})$; so we have only to prove that $q_{-2(q-r)}^{(j)}(x, y, \xi) \in S_{1,0}^{-2(q-r) p}(\Omega \times \mathbb{R}), j=1,2$.

First we obtain by induction on $n$ that for every $j, k \in \mathbb{Z}_{+}$there exists a constant $C_{j k}$ such that

$$
\left|D_{y}^{j} D_{\xi}^{k}\left(e^{-\tau \psi(y, \xi)} \partial_{\xi}^{n} e^{\tau \psi(y, \xi)}\right)\right| \leq C_{j k}(1+|\xi|)^{p r n-n-k} .
$$


Using the Leibniz rule and the estimate (3.9), it is easy to deduce that for every fixed $N, q_{-2(q-r)}^{(1)}(x, y, \xi) \in S_{1,0}^{-2(q-r) p}(\Omega \times \mathbb{R})$.

Now we consider $q_{-2(q-r)}^{(2)}(x, y, \xi)$. Let us observe that, $\psi(y, \xi)$ being essentially subadditive with respect to $\xi$, we get $e^{\tau \psi(y, \xi+t \eta)-\tau \psi(y, \xi)} \leq e^{\bar{C}(1+|\eta|)^{p r}}$; moreover, due to $\left|D_{y}^{j} D_{\xi}^{k} \psi(y, \xi)\right| \leq C_{j k}(1+|\xi|)^{p r-k}\left(\psi \in S_{1,0}^{r p}\right)$, and to the inequality $\frac{1}{1+|\xi+t \eta|} \leq$ $\frac{1+|\eta|}{1+|\xi|}$, we have that $\left|D_{y}^{j} D_{\xi}^{k}(\psi(y, \xi+t \eta)-\psi(y, \xi))\right| \leq \widetilde{C}_{j k}(1+|\xi|)^{p r-k}(1+|\eta|)^{p r+k}$. Indeed,

$$
\begin{aligned}
\mid D_{y}^{j} D_{\xi}^{k} & (\psi(y, \xi+t \eta)-\psi(y, \xi))|\leq| D_{y}^{j} D_{\xi}^{k}(\psi(y, \xi+t \eta))|+| D_{y}^{j} D_{\xi}^{k} \psi(y, \xi) \mid \\
& \leq C_{j k}(1+|\xi+t \eta|)^{p r-k}+C_{j k}(1+|\xi|)^{p r-k} \\
& \leq 2^{p r} C_{j k}(1+|\xi|)^{p r}(1+|\eta|)^{p r}\left(\frac{1+|\eta|}{1+|\xi|}\right)^{k}+C_{j k}(1+|\xi|)^{p r-k} \\
& \leq \widetilde{C}_{j k}(1+|\xi|)^{p r-k}(1+|\eta|)^{p r+k}
\end{aligned}
$$

So we obtain, using Faà di Bruno's estimate, that

$$
\begin{aligned}
& \left|D_{y}^{j} D_{\xi}^{k}\left(e^{\tau \psi(y, \xi+t \eta)-\tau \psi(y, \xi)}\right)\right| \leq \bar{C}_{j k} \sum_{0<h \leq j+k}\left|e^{\tau \psi(y, \xi+t \eta)-\tau \psi(y, \xi)}\right| \\
& \times \sum_{\substack{j_{1}+\ldots+j_{h}=j \\
k_{1}+\ldots+k_{h}=k}}\left|D_{y}^{j_{1}} D_{\xi}^{k_{1}}(\psi(y, \xi+t \eta)-\psi(y, \xi))\right| \cdots\left|D_{y}^{j_{h}} D_{\xi}^{k_{h}}(\psi(y, \xi+t \eta)-\psi(y, \xi))\right| \\
& \leq \widetilde{C}_{j k} e^{\bar{C}(1+|\eta|)^{p r}}(1+|\eta|)^{p r(j+k)+k}(1+|\xi|)^{p r(j+k)-k} .
\end{aligned}
$$

Using (3.9) with $\xi+t \eta$ instead of $\xi$, the previous estimate and the fact that $\frac{1}{1+|\xi+t \eta|} \leq \frac{1+|\eta|}{1+|\xi|}$, we have

$$
\begin{aligned}
& \left|D_{y}^{j} D_{\xi}^{k} r_{N}(y, \xi, \eta)\right| \\
& \quad \leq C_{j k}^{\prime}(1+|\eta|)^{p r(j+k)+p r N+N+k} e^{\bar{C}(1+|\eta|)^{p r}}(1+|\xi|)^{p r(j+k)+p r N-N-k} .
\end{aligned}
$$

Reasoning as in the proof of Theorem [2.2] we find that, if $a(x, y) \in G_{0}^{\left(q_{1}, q_{2}\right)}$, there exists a constant $M$ such that $\left|D_{y}^{j} \widetilde{a}(y, \eta)\right| \leq C_{j} e^{-M(1+|\eta|)^{p_{1}}}, p_{1}=\frac{1}{q_{1}}$. Using this fact, the estimate (3.10) and the Leibniz rule, we have

$$
\begin{aligned}
\mid D_{x}^{l} D_{y}^{j} D_{\xi}^{k} q_{-2(q-r)}^{(2)}( & x, y, \xi) \mid \leq C_{l j k}^{\prime}(1+|\xi|)^{p r(j+k)+p r N-N-k} \\
& \times \int(1+|\eta|)^{p r(j+k)+p r N+N+k}|\eta|^{l} e^{\bar{C}(1+|\eta|)^{p r}} e^{-M(1+|\eta|)^{p_{1}}} d \eta \\
\leq & C_{l j k}(1+|\xi|)^{p r(j+k)+p r N-N-k} ;
\end{aligned}
$$

since $p r<1$, taking $N$ sufficiently large, depending on $j$ and $k$, we obtain that the symbol $q_{-2(q-r)}(x, y, \xi)=q_{-2(q-r)}^{(1)}(x, y, \xi)+q_{-2(q-r)}^{(2)}(x, y, \xi)$ is in the class $S_{1,0}^{-2(q-r) p}(\Omega \times \mathbb{R})$.

Definition 3.1. Let us consider a function $a(x, y, \xi, \eta) \in C^{\infty}\left(\Omega \times \mathbb{R}^{2}\right)$ and define

$$
\lambda_{p}(\xi, \eta)=\left(1+|\xi|^{2 p}+|\eta|^{2}\right)^{\frac{1}{2}} \sim 1+|\xi|^{p}+|\eta| .
$$

We say that $a(x, y, \xi, \eta) \in S_{(p, 1)}^{m, \mu}\left(\Omega \times \mathbb{R}^{2}\right), p \leq 1$, if for every $l, j, k, h \in \mathbb{Z}_{+}$there exists a constant $C_{l j k h}$ such that

$$
\left|D_{x}^{l} D_{y}^{j} D_{\xi}^{k} D_{\eta}^{h} a(x, y, \xi, \eta)\right| \leq C_{l j k h} \lambda_{p}(\xi, \eta)^{m}(1+|\xi|)^{p \mu-k-p h} .
$$


Proposition 3.2. Let $p, r$, the function $a(x, y)$ and the weight function $\psi(y, \xi)$ be fixed as in Proposition [3.1. Then

$$
\begin{aligned}
e^{\tau \psi\left(y, D_{x}\right)} a(x, y) D_{x}^{l} D_{y}^{j} e^{-\tau \psi\left(y, D_{x}\right)} & \\
= & a(x, y) D_{x}^{l} D_{y}^{j}-j \tau a(x, y)\left(D_{y} \psi\right)\left(y, D_{x}\right) D_{x}^{l} D_{y}^{j-1} \\
& +A_{\frac{l}{p}+j, \max \{-2(1-r),-(q-r)\}}\left(x, y, D_{x}, D_{y}\right),
\end{aligned}
$$

where the symbol $a_{\frac{l}{p}+j, \max \{-2(1-r),-(q-r)\}}(x, y, \xi, \eta)$ of the pseudo-differential operator $A_{\frac{l}{p}+j, \max \{-2(1-r),-(q-r)\}}\left(x, y, D_{x}, D_{y}\right)$ belongs to the class

$$
S_{(p, 1)}^{\frac{l}{p}+j, \max \{-2(1-r),-(q-r)\}}\left(\Omega \times \mathbb{R}^{2}\right) .
$$

Remark 3.1. The operator $-j \tau a(x, y)\left(D_{y} \psi\right)\left(y, D_{x}\right) D_{x}^{l} D_{y}^{j-1}$ in (3.13) has the symbol $-j \tau a(x, y)\left(D_{y} \psi\right)(y, \xi) \xi^{l} \eta^{j-1}$ in the class $S_{(p, 1)}^{\frac{l}{p}+j,-(1-r)}\left(\Omega \times \mathbb{R}^{2}\right)$, as is easy to prove since $\psi \in S_{1,0}^{r p}$.

Proof. Using the identities (2.2)-(2.4) and Proposition 3.1, we get

$$
\begin{aligned}
& e^{\tau \psi\left(y, D_{x}\right)} a(x, y) D_{x}^{l} D_{y}^{j} e^{-\tau \psi\left(y, D_{x}\right)} \\
&=e^{\tau \psi\left(y, D_{x}\right)} a(x, y) e^{-\tau \psi\left(y, D_{x}\right)} e^{\tau \psi\left(y, D_{x}\right)} D_{x}^{l} D_{y}^{j} e^{-\tau \psi\left(y, D_{x}\right)} \\
&=\left(a(x, y)+Q_{-(q-r)}\left(x, y, D_{x}\right)+Q_{-2(q-r)}\left(x, y, D_{x}\right)\right) \\
& \times\left(D_{x}^{l} D_{y}^{j}-j \tau\left(D_{y} \psi\right)\left(y, D_{x}\right) D_{x}^{l} D_{y}^{j-1}+\sum_{h=0}^{j-2} q_{j-h}^{(j)}\left(y, D_{x}\right) D_{x}^{l} D_{y}^{h}\right) \\
&= a(x, y) D_{x}^{l} D_{y}^{j}-j \tau a(x, y)\left(D_{y} \psi\right)\left(y, D_{x}\right) D_{x}^{l} D_{y}^{j-1} \\
&+A_{\frac{l}{p}+j, \max \{-2(1-r),-(q-r)\}}\left(x, y, D_{x}, D_{y}\right) .
\end{aligned}
$$

In the expression of the symbol of the last operator, let us analyze, for example, the term $a(x, y) q_{j-h}^{(j)}(y, \xi) \xi^{l} \eta^{h}$, for $h=0, \ldots, j-2$. We get, for $l^{\prime}, j^{\prime}, k^{\prime} \in \mathbb{Z}_{+}$and $h^{\prime} \leq h$,

$$
\begin{aligned}
& \left|D_{x}^{l^{\prime}} D_{y}^{j^{\prime}} D_{\xi}^{k^{\prime}} D_{\eta}^{h^{\prime}}\left(a(x, y) q_{j-h}^{(j)}(y, \xi) \xi^{l} \eta^{h}\right)\right| \\
& \leq C_{l^{\prime} j^{\prime} k^{\prime} h^{\prime}}^{\prime} \sum_{j_{1}^{\prime}+j_{2}^{\prime}=j^{\prime}} \sum_{\substack{1 \\
k_{1}^{\prime}+k_{2}^{\prime}=k^{\prime} \\
k_{2}^{\prime} \leq l}}\left|D_{x}^{l^{\prime}} D_{y}^{j_{1}^{\prime}} a(x, y)\right|\left|D_{y}^{j_{2}^{\prime}} D_{\xi}^{k_{1}^{\prime}} q_{j-h}^{(j)}(y, \xi)\right||\xi|^{l-k_{2}^{\prime}}|\eta|^{h-h^{\prime}} \\
& \leq C_{l^{\prime} j^{\prime} k^{\prime} h^{\prime}}(1+|\xi|)^{p r(j-h)-k_{1}^{\prime}}(1+|\xi|)^{l-k_{2}^{\prime}} \lambda_{p}(\xi, \eta)^{h-h^{\prime}} \\
& \leq C_{l^{\prime} j^{\prime} k^{\prime} h^{\prime}} \lambda_{p}(\xi, \eta)^{\frac{l}{p}+j-2(1-r)-h^{\prime}}(1+|\xi|)^{-k^{\prime}} \\
& \leq C_{l^{\prime} j^{\prime} k^{\prime} h^{\prime}} \lambda_{p}(\xi, \eta)^{\frac{l}{p}+j}(1+|\xi|)^{-2 p(1-r)-k^{\prime}-p h^{\prime}}
\end{aligned}
$$

In general, using the Leibniz rule, the estimates on $\psi \in S_{1,0}^{r p}$ and $q_{j-h} \in S_{1,0}^{p r(j-h)}$, and the fact that the symbols of the operators $q_{-k(q-r)}\left(x, y, D_{x}\right)$ are in the class $S_{1,0}^{-k(q-r) p}(\Omega \times \mathbb{R})$ for $k=1,2$, we obtain:

- $\left(a(x, y)+q_{-(q-r)}(x, y, \xi)+q_{-2(q-r)}(x, y, \xi)\right) \sum_{h=0}^{j-2} q_{j-h}^{(j)}(y, \xi) \xi^{l} \eta^{h}$ is in the class $S_{(p, 1)}^{\frac{l}{p}+j,-2(1-r)}\left(\Omega \times \mathbb{R}^{2}\right) ;$ 
- $\left(q_{-(q-r)}(x, y, \xi)+q_{-2(q-r)}(x, y, \xi)\right)\left(\xi^{l} \eta^{j}-j \tau\left(D_{y} \psi\right)(y, \xi) \xi^{l} \eta^{j-1}\right)$ is in the class $S_{(p, 1)}^{\frac{l}{p}+j,-(q-r)}\left(\Omega \times \mathbb{R}^{2}\right)$.

Let us observe that $S_{(p, 1)}^{\frac{l}{p}+j,-2(1-r)}\left(\Omega \times \mathbb{R}^{2}\right)$ and $S_{(p, 1)}^{\frac{l}{p}+j,-(q-r)}\left(\Omega \times \mathbb{R}^{2}\right)$ are both contained in $S_{(p, 1)}^{\frac{l}{p}+j, \max \{-2(1-r),-(q-r)\}}\left(\Omega \times \mathbb{R}^{2}\right)$; so it follows immediately that $a_{\frac{l}{p}+j, \max \{-2(1-r),-(q-r)\}}(x, y, \xi, \eta) \in S_{(p, 1)}^{\frac{l}{p}+j, \max \{-2(1-r),-(q-r)\}}\left(\Omega \times \mathbb{R}^{2}\right)$.

Now we want to analyze the behavior of the conjugate operator, defined by (3.4). We choose the weight function in the following way:

$$
\psi(y, \xi)=\left(1+\frac{y}{2 \delta}\right) \varphi(\xi)|\xi|^{\frac{d}{m} r} \quad\left(\psi(y, \xi)=\left(1-\frac{y}{2 \delta}\right) \varphi(\xi)|\xi|^{\frac{d}{m} r}\right),
$$

where $0<r<1, \delta>0$ and $\varphi(\xi)$ is a $C^{\infty}$ function such that $0 \leq \varphi(\xi) \leq 1, \varphi(\xi)=0$ for $|\xi| \leq \frac{1}{2}$ and $\varphi(\xi)=1$ for $|\xi| \geq 1$. Observe that $\psi(y, \xi)$ is a weight function of order $\left(r, \frac{d}{m}\right)$, essentially subadditive with respect to $\xi$.

Proposition 3.3. Let us fix the operator $P\left(x, y, D_{x}, D_{y}\right)$ as in (1.2) with $d<m$, and let us fix $0<r<1$; we choose the weight function as in (3.14). Then the symbol of the conjugate operator is given by

$$
\begin{aligned}
\widetilde{p}(x, y, \xi, \eta) & =p(x, y, \xi, \eta)-i \frac{m \tau}{2 \delta} \varphi(\xi)|\xi|^{\frac{d}{m} r} \eta^{m-1}+p_{m,-(1-r)-\nu}(x, y, \xi, \eta) \\
(\widetilde{p}(x, y, \xi, \eta) & \left.=p(x, y, \xi, \eta)+i \frac{m \tau}{2 \delta} \varphi(\xi)|\xi|^{\frac{d}{m} r} \eta^{m-1}+p_{m,-(1-r)-\nu}(x, y, \xi, \eta)\right),
\end{aligned}
$$

where $p(x, y, \xi, \eta)$ is the symbol of $P$ and

$$
p_{m,-(1-r)-\nu}(x, y, \xi, \eta) \in S_{\left(\frac{d}{m}, 1\right)}^{m,-(1-r)-\nu}\left(\Omega \times \mathbb{R}^{2}\right), \nu>0 .
$$

Observe that

$$
\left(i \frac{m \tau}{2 \delta} \varphi(\xi)|\xi|^{\frac{d}{m} r} \eta^{m-1}\right) \in S_{\left(\frac{d}{m}, 1\right)}^{m,-(1-r)}\left(\Omega \times \mathbb{R}^{2}\right) .
$$

Proof. First we observe that we can write the operator $P\left(x, y, D_{x}, D_{y}\right)$ in the following way:

$$
P\left(x, y, D_{x}, D_{y}\right)=D_{y}^{m}-b_{0}(x, y) D_{x}^{d}+\sum_{m-t \leq l} \sum_{\frac{m}{d}+j \leq m-\epsilon_{0}} a_{l j}(x, y) D_{x}^{l} D_{y}^{j},
$$

where $\epsilon_{0}$ is given by with $t=0$.

Now, applying Proposition 3.1 and Proposition 3.2 with $p=\frac{d}{m}$ and $\psi$ as in (3.14), and using (2.4), we get

$$
\begin{aligned}
\widetilde{P}\left(x, y, D_{x}, D_{y}\right)=e^{\tau \psi\left(y, D_{x}\right)} D_{y}^{m} e^{-\tau \psi\left(y, D_{x}\right)}-e^{\tau \psi\left(y, D_{x}\right)} b_{0}(x, y) e^{-\tau \psi\left(y, D_{x}\right)} D_{x}^{d} \\
\quad+\sum_{m-t \leq l \frac{m}{d}+j \leq m-\epsilon_{0}} e^{\tau \psi\left(y, D_{x}\right)} a_{l j}(x, y) D_{x}^{l} D_{y}^{j} e^{-\tau \psi\left(y, D_{x}\right)} \\
=D_{y}^{m}-m \tau\left(D_{y} \psi\right)\left(y, D_{x}\right) D_{y}^{m-1}+A_{m, \max \left\{-2(1-r),-\left(\frac{m}{d}-r\right)\right\}}\left(x, y, D_{x}, D_{y}\right) \\
\quad-b_{0}(x, y) D_{x}^{d}-Q_{-\left(\frac{m}{d}-r\right)}\left(x, y, D_{x}\right) D_{x}^{d} \\
\quad+\sum_{m-t \leq l \frac{m}{d}+j \leq m-\epsilon_{0}} a_{l j}(x, y) D_{x}^{l} D_{y}^{j}+A_{m,-(1-r)-\epsilon_{0}}^{\prime}\left(x, y, D_{x}, D_{y}\right) \\
\quad \\
\quad P\left(x, y, D_{x}, D_{y}\right)-m \tau\left(D_{y} \psi\right)\left(y, D_{x}\right) D_{y}^{m-1}+P_{m,-(1-r)-\nu}\left(x, y, D_{x}, D_{y}\right),
\end{aligned}
$$


where

$$
\begin{gathered}
a_{m, \max \left\{-2(1-r),-\left(\frac{m}{d}-r\right)\right\}}(x, y, \xi, \eta) \in S_{\left(\frac{d}{m}, 1\right)}^{m, \max \left\{-2(1-r),-\left(\frac{m}{d}-r\right)\right\}}\left(\Omega \times \mathbb{R}^{2}\right), \\
q_{-\left(\frac{m}{d}-r\right)}(x, y, \xi) \in S_{1,0}^{-\left(\frac{m}{d}-r\right) \frac{d}{m}}(\Omega \times \mathbb{R}) \\
a_{m,-(1-r)-\epsilon_{0}}^{\prime}(x, y, \xi, \eta) \in S_{\left(\frac{d}{m}, 1\right)}^{m,-(1-r)-\epsilon_{0}}\left(\Omega \times \mathbb{R}^{2}\right) .
\end{gathered}
$$

By Definition 3.1 we can deduce that the symbol $p_{m,-(1-r)-\nu}(x, y, \xi, \eta)=$ $a_{m, \max \left\{-2(1-r),-\left(\frac{m}{d}-r\right)\right\}}(x, y, \xi, \eta)-q_{-\left(\frac{m}{d}-r\right)}(x, y, \xi) \xi^{d}+a_{m,-(1-r)-\epsilon_{0}}^{\prime}(x, y, \xi, \eta)$ is in the class $S_{\left(\frac{d}{m}, 1\right)}^{m,-(1-r)-\nu}\left(\Omega \times \mathbb{R}^{2}\right)$, where $\nu=\min \left\{1-r, \frac{m}{d}-1, \epsilon_{0}\right\}$. We observe that, since $r<1$ and $d<m$, we have $\nu>0$.

The following theorem allows us to find a parametrix of the operator (1.2) when $m-t=\frac{k^{*}}{d}$, for a positive integer $k^{*}$ such that $d\left(m-\frac{1}{2}\right)<k^{*}<d m$ and the set $I_{k^{*}}:=\left\{(l, j) \in \mathbb{Z}_{+}^{2}: d j+m l=k^{*}\right\}$ consists of just one couple $\left(l^{*}, j^{*}\right)$. We take $b_{0}(x, y)$ and $a_{l j}(x, y)$ in $C^{\infty}(\Omega)$.

Theorem 3.1 (Sobolev parametrix). Assume that 1.5) in Theorem 1.1 and (1.3) hold. Then there exists a linear map

$$
E_{\infty}: H_{\frac{d}{m}}^{s}(\mathbb{R} \times(-\delta, \delta)) \longrightarrow H_{\frac{d}{m}}^{s+\frac{k^{*}}{d}}(\mathbb{R} \times(-\delta, \delta))
$$

such that

$$
P\left(x, y, D_{x}, D_{y}\right) E_{\infty} u=\vartheta(x, y) u+R_{\infty} u,
$$

where $\vartheta(x, y) \in C^{\infty}, \vartheta(x, y)=1$ in a neighborhood of the origin and $R_{\infty}$ is a regularizing map in the Sobolev anisotropic spaces.

Removing hypothesis $i$ ) in Theorem 1.1, we shall prove that there exists a Gevrey-Sobolev parametrix of the operator (1.2) for $t<\frac{1}{2}$, where now we take analytic coefficients, or more generally coefficients in the anisotropic Gevrey space $G_{0}^{\left(q_{1}, q_{2}\right)}(\Omega), 1<q_{1}<\frac{m}{d r}, q_{2}>1$ (cf. Proposition 3.1), with $r>\max \left\{\frac{1}{2}, 1-t-\epsilon_{t}\right\}$.

Theorem 3.2 (Gevrey-Sobolev parametrix). Let one of the conditions [a] or [b] in Theorem 1.2 and (1.3) hold. Then there exists a linear map

$$
E: \mathbb{H}_{\tau, \frac{m}{d}, r}^{s, \psi}(\mathbb{R} \times(-\delta, \delta)) \longrightarrow \mathbb{H}_{\tau, \frac{m}{d}, r}^{s+m-(1-r), \psi}(\mathbb{R} \times(-\delta, \delta))
$$

such that

$$
P\left(x, y, D_{x}, D_{y}\right) E u=\chi(x, y) u+R u,
$$

where $\chi(x, y) \in G_{0}^{\left(q_{1}, q_{2}\right)}(\Omega), \chi(x, y)=1$ in a neighborhood of the origin, and $R$ is a regularizing map in the anisotropic Gevrey-Sobolev spaces.

The proof of Theorems 3.1 and 3.2 will be deduced from Theorem 3.3 . below, about the hypoellipticity of the following class of differential polynomials with $C^{\infty}$ coefficients $h_{(\cdot, \cdot)}: \Omega \rightarrow \mathbb{C}$ :

$$
p(x, y, \xi, \eta)=\eta^{m}-h_{d 0}(x, y) \xi^{d}+\sum_{(l, j) \in I} h_{l j}(x, y) \xi^{l} \eta^{j}+\sigma(z, \zeta),
$$

for $\zeta=(\xi, \eta)$ the dual variable of $z=(x, y)$. We define the following sets for $k \in \mathbb{R}_{+}, 0<k<d m$ :

$$
I_{k}=\left\{(l, j) \in \mathbb{R}_{+} \times \mathbb{Z}_{+}: d j+m l=k\right\}
$$


and fix $k=k^{*}$ such that $d\left(m-\frac{1}{2}\right)<k^{*}<d m$. We use the notation $k^{-}$for all $k<k^{*}$ and $k^{+}$for all $k>k^{*}$. We define $I=I_{-} \cup I_{k^{*}} \cup I_{+}$, with $I_{-}=\bigcup I_{k^{-}}$, $I_{+}=\bigcup I_{k^{+}}$. The symbol $\sigma(x, y, \xi, \eta)$ in $C^{\infty}\left(\mathbb{R}^{2} \times \mathbb{R}^{2}\right)$ is such that

$$
\left|D_{x}^{\alpha} D_{y}^{\beta} D_{\xi}^{\gamma} D_{\eta}^{\theta} \sigma(z, \zeta)\right| \leq C_{\alpha \beta \gamma \theta}(1+\lambda(\zeta))^{\bar{m}-\left(\gamma \frac{m}{d}+\theta\right)}, \quad \bar{m}<\frac{k^{*}}{d},
$$

where $\lambda(\zeta)=|\xi|^{\frac{d}{m}}+|\eta|$ is the anisotropic norm; cf. the expression of the anisotropic Sobolev spaces in Definition $2.2 H_{\left(\frac{d}{m}, 1\right)}^{s}\left(\mathbb{R}^{2}\right), s \geq 0$. We recall that $\Sigma:=\{(z, \zeta) \in$ $\left.\Omega \times \mathbb{R}^{2} \backslash\{0\}: \eta^{m}-\Re b_{d 0} \xi^{d}=0\right\}$ is the anisotropic characteristic manifold of $p(x, y, \xi, \eta)$ in (3.16); letting $\Lambda$ be a neighborhood of $\Sigma$, we denote by $\Gamma$ the set $\Omega \times \Lambda$, and we state the following:

Theorem 3.3. Assume that $I_{k^{*}}$ consists of just one couple $\left(l^{*}, j^{*}\right), k^{*}=d j^{*}+m l^{*}$, such that:

i) $\Im h_{l^{*} j^{*}}(x, y) \neq 0$ for all $(x, y) \in \Omega$,

ii) for all $(l, j)$ such that $d j+m l>k^{*}=d j^{*}+m l^{*}$,

$$
\begin{aligned}
& \Im h_{l^{*} j^{*}}(x, y) \Im h_{l j}(x, y) \eta^{j+j^{*}} \xi^{l+l^{*}} \geq 0, \quad(z, \zeta) \in \Gamma, \\
& \text { iii) } \Im h_{l^{*} j^{*}}(x, y) \Im h_{d 0}(x, y) \eta^{j^{*}} \xi^{d+l^{*}} \leq 0, \quad(z, \zeta) \in \Gamma, \\
& \text { iv) } \Re h_{d 0}(x, y) \neq 0, \text { for all }(x, y) \in \Omega .
\end{aligned}
$$

Then

$$
|p(x, y, \xi, \eta)| \geq b \lambda(\zeta)^{\frac{k^{*}}{d}} \text { in } \Omega \times \mathbb{R}^{2},
$$

for a suitable constant $b$. Also, for all $(\alpha, \beta) \in \mathbb{Z}_{+}^{2},(\gamma, \theta) \in \mathbb{Z}_{+}^{2}$ and for all $K \subset \subset \Omega$ we have, with suitable constants $L_{\alpha, \beta, \gamma, \theta}$ and $B$, that

$$
\frac{\left|D_{x}^{\alpha} D_{y}^{\beta} D_{\xi}^{\gamma} D_{\eta}^{\theta} p(x, y, \xi, \eta)\right| \lambda(\zeta)^{\rho\left(\gamma \frac{m}{d}+\theta\right)-\delta\left(\alpha \frac{m}{d}+\beta\right)}}{|p(x, y, \xi, \eta)|} \leq L_{\alpha, \beta, \gamma, \theta},|\xi|+|\eta|>B,
$$

with $\rho=\frac{k^{*}-d(m-1)}{d}, \delta=\frac{d m-k^{*}}{d}$. Observe that $\delta<\rho$, since we have assumed $k^{*}>d\left(m-\frac{1}{2}\right)$.

Remark 3.2. By formula (3.20) and by Mascarello and Rodino (MR], Theorem 3.3.6), we have that the operator $P(z, D)$, associated to the symbol $p(z, \zeta)$ in (3.16), is $C^{\infty}$-microlocally hypoelliptic in $\Gamma$; i.e., $\Gamma \cap W F P u=\Gamma \cap W F u$, for all $u \in$ $\mathcal{D}^{\prime}(\Omega)$, where $W F u$ is the Hörmander wave front set. A microhypoelliptic operator is hypoelliptic too. If the coefficients are analytic and (3.17) holds for $C_{\alpha \beta \gamma \theta}=$ $L^{\alpha+\beta+\gamma+\theta+1} \alpha ! \beta ! \gamma ! \theta !$, we obtain $G^{\sigma}$-hypoellipticity, $\sigma \geq \frac{d}{k^{*}-d(m-1)}$.

Before starting the proof of Theorems 3.1 and 3.2 we also need the following class of symbols:

Definition 3.2. Let $a(x, y, \xi, \eta) \in C^{\infty}\left(\Omega \times \mathbb{R}^{2}\right)$. We say that $a(x, y, \xi, \eta) \in$ $S_{1,0}^{m ;(p, 1)}\left(\Omega \times \mathbb{R}^{2}\right)$ if for every $l, j, k, h \in \mathbb{Z}_{+}$there exists a constant $C_{l j k h}$ such that

$$
\left|D_{x}^{l} D_{y}^{j} D_{\xi}^{k} D_{\eta}^{h} a(x, y, \xi, \eta)\right| \leq C_{l j k h} \lambda_{p}(\xi, \eta)^{m-\frac{k}{p}-h},
$$

where $\lambda_{p}(\xi, \eta)$ is given by (3.11). 
Further on we shall also refer to the microlocal classes of symbols $S_{(p, 1)}^{m, \mu}(\Gamma)$, $S_{1,0}^{m ;(p, 1)}(\Gamma)$, where now $\Gamma$ is a quasi-homogeneous cone. We leave to the reader the obvious definitions in this frame.

In the following we suppose that the quasi-homogeneous cone $\Gamma$ is included in a region in which $|\xi|^{p} \geq c_{0}|\eta|$ (in particular we are interested in the case $p=\frac{d}{m}$ ); for these sets we have that $(1+|\xi|)^{p} \sim \lambda_{p}(\xi, \eta)$, and so $S_{(p, 1)}^{m, \mu}(\Gamma)$ can be identified with $S_{1,0}^{m+\mu ;(p, 1)}(\Gamma)$.

Definition 3.3. Let $q(x, y, \xi, \eta) \in S_{1,0}^{m ;(p, 1)}(\Gamma)$. Let us suppose that $m^{\prime} \leq m$ and $0 \leq \delta<\rho \leq 1$. We say that $q(x, y, \xi, \eta)$ is of type $\left(m, m^{\prime}, p, \rho, \delta\right)$ if there exist positive constants $c, C, C_{l j k h}$ such that in $\Gamma$ the following estimates hold:

$$
\begin{gathered}
|q(x, y, \xi, \eta)| \geq c \lambda_{p}(\xi, \eta)^{m^{\prime}} \\
\left|D_{x}^{l} D_{y}^{j} D_{\xi}^{k} D_{\eta}^{h} q(x, y, \xi, \eta)\right| \leq C_{l j k h}|q(x, y, \xi, \eta)| \lambda_{p}(\xi, \eta)^{-\rho\left(\frac{k}{p}+h\right)+\delta\left(\frac{l}{p}+j\right)}
\end{gathered}
$$

for $\lambda_{p}(\xi, \eta) \geq C$.

Proposition 3.4. Let $q(x, y, \xi, \eta)$ be of the type $\left(m, m^{\prime}, p, \rho, \delta\right)$. Then there exists a symbol $q^{\prime}(x, y, \xi, \eta) \in S_{\rho, \delta}^{-m^{\prime} ;(p, 1)}(\Gamma)$, i.e.,

$$
\left|D_{x}^{l} D_{y}^{j} D_{\xi}^{k} D_{\eta}^{h} q^{\prime}(x, y, \xi, \eta)\right| \leq C_{l j k h} \lambda_{p}(\xi, \eta)^{m-\rho\left(\frac{k}{p}+h\right)+\delta\left(\frac{l}{p}+j\right)},
$$

$q^{\prime}$ being the parametrix of $q(x, y, \xi, \eta)$, i.e., $q \# q^{\prime} \sim q^{\prime} \# q \sim 1$, where \# indicates the standard asymptotic product.

For the proof of this proposition, see Hunt and Piriou $\mathrm{HP}$.

Proof of Theorem 3.1 (Sobolev parametrix). Without loss of generality, we may assume $\rho^{0}=\left(\xi_{0}, \eta_{0}\right)$ with $\xi_{0}>0$. The quasi-homogeneous conic neighborhood $\Gamma$ of $\rho^{0}$ is then included in a region $\left\{\xi>0, \eta^{2}<C \xi^{2}\right\}$. Since $P$ satisfies all the hypotheses of Theorem 3.3 , we have that the symbol $p(x, y, \xi, \eta)$ is of the type $\left(m, \frac{k^{*}}{d}, \frac{d}{m}, \frac{k^{*}-d(m-1)}{d}, \frac{d m-k^{*}}{d}\right)$. So using Proposition 3.4 we can find a linear map $E: H_{\frac{d}{m}}^{s} \longrightarrow H_{\frac{d}{m}}^{s+\frac{k^{*}}{d}}$ such that $P E=\vartheta(x, y) \varrho\left(D_{x}, D_{y}\right)+R$, where $\vartheta(x, y) \in C_{0}^{\infty}(\Omega)$, $\varrho(\xi, \eta) \in C^{\infty}\left(\mathbb{R}^{2}\right)$ with support in a quasi-homogeneous conic neighborhood of $\left(\xi_{0}, \eta_{0}\right), R: H_{\frac{d}{m}}^{s}(\mathbb{R} \times(-\delta, \delta)) \longrightarrow H_{\frac{d}{m}}^{t}(\mathbb{R} \times(-\delta, \delta))$ for every $t \geq 0$. So we can construct two operators $E_{1}$ and $R_{1}$ such that $P E_{1}=\vartheta(x, y) \varrho\left(D_{x}, D_{y}\right)+R_{1}$, where we can choose the function $\varrho(\xi, \eta) \in C^{\infty}$ quasi-homogeneous in $(\xi, \eta)$ of order $\left(\frac{d}{m}, 1\right)$ and of degree 0 for large $\left(|\xi|^{\frac{d}{m}}+|\eta|\right), \varrho(\xi, \eta)=0$ in a quasi-homogeneous conic neighborhood of $\xi=0$ and $R_{1}: H_{\frac{d}{m}}^{s}(\mathbb{R} \times(-\delta, \delta)) \longrightarrow H_{\frac{d}{m}}^{t}(\mathbb{R} \times(-\delta, \delta))$ for every $t \geq 0$. We can suppose that $\operatorname{supp}(1-\varrho(\xi, \eta)) \subset \Gamma_{0}$, where $\Gamma_{0}$ is a sufficiently small neighborhood of $\xi=0$ such that $\eta^{m}-b_{0}(x, y) \xi^{d}$ is quasi-elliptic in $\Gamma_{0}$. In the following we denote by $\bar{p}_{m}(x, y, \xi, \eta)$ the anisotropic principal symbol of $P\left(x, y, D_{x}, D_{y}\right)$, i.e., $\bar{p}_{m}(x, y, \xi, \eta)=\eta^{m}-b_{0}(x, y) \xi^{d}$. By the results of Hunt and Piriou [HP] we have, in $\Gamma_{0}$,

$$
\begin{aligned}
p(x, y, \xi, \eta) \# \bar{p}_{m}^{-1}(x, y, \xi, \eta) & \\
& =\left(\bar{p}_{m}(x, y, \xi, \eta)+q_{m-\epsilon_{m}}(x, y, \xi, \eta)\right) \# \bar{p}_{m}^{-1}(x, y, \xi, \eta) \\
& =1+q_{-\epsilon_{m}}(x, y, \xi, \eta),
\end{aligned}
$$


where $q_{-\epsilon_{m}} \in S_{0,0}^{-\epsilon_{m} ;\left(\frac{d}{m}, 1\right)}$ and $\epsilon_{m}$ is given by (3.1). In a standard way we can construct $s_{-\epsilon_{m}} \in S_{0,0}^{-\epsilon_{m} ;\left(\frac{d}{m}, 1\right)}$ such that

$$
\left(1+q_{-\epsilon_{m}}(x, y, \xi, \eta)\right) \#\left(1+s_{-\epsilon_{m}}(x, y, \xi, \eta)\right) \sim 1 .
$$

Let us consider now the symbol

$$
e_{0}=\bar{p}_{m}^{-1}(x, y, \xi, \eta) \#\left(1+s_{-\epsilon_{m}}(x, y, \xi, \eta)\right) \# \vartheta(x, y)(1-\varrho(\xi, \eta))
$$

and let $E_{0}$ be the pseudo-differential operator of symbol $e_{0}$. By construction we obtain $P E_{0}=\vartheta(x, y)\left(1-\varrho\left(D_{x}, D_{y}\right)\right)+R_{0}$, with $R_{0}$ regularizing on the anisotropic Sobolev spaces $H_{\frac{d}{m}}^{s}(\mathbb{R} \times(-\delta, \delta))$. Taking $E_{\infty}=E_{1}+E_{0}$, we have that

$$
P E_{\infty}=P E_{1}+P E_{0}=\vartheta(x, y)+R_{\infty},
$$

where $R_{\infty}=R_{1}+R_{0}$ is regularizing on $H_{\frac{d}{m}}^{s}(\mathbb{R} \times(-\delta, \delta))$.

Proof of Theorem 3.2 (Gevrey-Sobolev parametrix). Let us suppose that one of the global conditions [a] or [b] holds. When [a] is satisfied we fix the weight function as in (3.14); if [b] holds we choose $\psi(y, \xi)=\left(1+\frac{y}{2 \delta} \operatorname{sign} \xi\right) \varphi(\xi)|\xi|^{\frac{d}{m} r}(\psi(y, \xi)=(1-$ $\left.\left.\frac{y}{2 \delta} \operatorname{sign} \xi\right) \varphi(\xi)|\xi|^{\frac{d}{m} r}\right)$. By Proposition 3.3, the symbol $\widetilde{p}(x, y, \xi, \eta)$ of the conjugate operator $\widetilde{P}\left(x, y, D_{x}, D_{y}\right)$ defined by (3.4) satisfies all the hypotheses of Theorem 3.3 with $j^{*}=m-1, l^{*}=\frac{d}{m} r$. So $\widetilde{p}(x, y, \xi, \eta)$ is of type $(m, m-(1-r), p, r, 1-r)$. As in the first part of the proof of Theorem 3.1, by Proposition [3.4 we can find a linear map

$$
\widetilde{E}: H_{\frac{d}{m}}^{s}(\mathbb{R} \times(-\delta, \delta)) \longrightarrow H_{\frac{d}{m}}^{s+m-(1-r)}(\mathbb{R} \times(-\delta, \delta))
$$

such that

$$
\widetilde{P} \widetilde{E}=\chi(x, y) \kappa\left(D_{x}, D_{y}\right)+\widetilde{R},
$$

where $\chi(x, y)$ is arbitrarily fixed in $C_{0}^{\infty}(\Omega), \kappa(\xi, \eta)$ is arbitrarily fixed in $C^{\infty}\left(\mathbb{R}^{2}\right)$ with support in a quasi-homogeneous conic neighborhood of $\left(\xi_{0}, \eta_{0}\right)$, and $\widetilde{R}$ is a regularizing operator in the anisotropic Sobolev spaces $H_{\frac{d}{m}}^{s}$. So we can find $\widetilde{E}_{1}$ and $\widetilde{R}_{1}$ such that $\widetilde{P} \widetilde{E}_{1}=\chi(x, y) \kappa\left(D_{x}, D_{y}\right)+\widetilde{R}_{1}$, where we can choose the $C^{\infty}$ function $\kappa(\xi, \eta)$ with the properties of the function $\varrho(x, y)$ in the previous proof $(\kappa(\xi, \eta)$ quasi-homogeneous of degree 0 out of the origin, $\kappa(\xi, \eta)=0$ in a neighborhood of $\xi=0$ and $\operatorname{supp}(1-\kappa(\xi, \eta)) \subset \Gamma_{0}$, where $\bar{p}_{m}(x, y, \xi, \eta)$ is quasi-elliptic in $\left.\Gamma_{0}\right)$; moreover, $\widetilde{R}_{1}: H_{\frac{d}{m}}^{s}(\mathbb{R} \times(-\delta, \delta)) \longrightarrow H_{\frac{d}{m}}^{t}(\mathbb{R} \times(-\delta, \delta))$ for every $t \geq 0$.

Reasoning as in the preceding proof and using Proposition 3.3 and the results of Hunt and Piriou $\left[\mathrm{HP}\right.$, we have, in $\Gamma_{0}$,

$$
\begin{aligned}
\widetilde{p}(x, y, \xi, \eta) \# \bar{p}_{m}^{-1}(x, y, \xi, \eta) & \\
& =\left(\bar{p}_{m}(x, y, \xi, \eta)+q_{m-\min \left\{\epsilon_{m}, 1-r\right\}}(x, y, \xi, \eta)\right) \# \bar{p}_{m}^{-1}(x, y, \xi, \eta) \\
& =1+q_{-\min \left\{\epsilon_{m}, 1-r\right\}}(x, y, \xi, \eta),
\end{aligned}
$$

where the symbol $q_{-\min \left\{\epsilon_{m}, 1-r\right\}}$ is in the class $S_{0,0}^{-\min \left\{\epsilon_{m}, 1-r\right\} ;\left(\frac{d}{m}, 1\right)}$. In a standard way we can construct $s_{-\min \left\{\epsilon_{m}, 1-r\right\}} \in S_{0,0}^{-\min \left\{\epsilon_{m}, 1-r\right\} ;\left(\frac{d}{m}, 1\right)}$ such that

$$
\left(1+q_{-\min \left\{\epsilon_{m}, 1-r\right\}}(x, y, \xi, \eta)\right) \#\left(1+s_{-\min \left\{\epsilon_{m}, 1-r\right\}}(x, y, \xi, \eta)\right) \sim 1 .
$$

Now let us consider the pseudo-differential operator $\widetilde{E}_{2}$ of the symbol

$$
\widetilde{e}_{2}=\bar{p}_{m}^{-1}(x, y, \xi, \eta) \#\left(1+s_{-\min \left\{\epsilon_{m}, 1-r\right\}}(x, y, \xi, \eta)\right) \# \chi(x, y)(1-\kappa(\xi, \eta)) .
$$


By construction we obtain $\widetilde{P} \widetilde{E}_{2}=\chi(x, y)\left(1-\kappa\left(D_{x}, D_{y}\right)\right)+\widetilde{R}_{2}$ with $\widetilde{R}_{2}$ regularizing on the anisotropic Sobolev spaces $H_{\frac{d}{m}}^{s}(\mathbb{R} \times(-\delta, \delta))$. Let $\widetilde{E}=\widetilde{E}_{1}+\widetilde{E}_{2}$. Then $\widetilde{P} \widetilde{E}=\widetilde{P} \widetilde{E}_{1}+\widetilde{P} \widetilde{E}_{2}=\chi(x, y)+\widetilde{R}, \widetilde{R}$ regularizing.

After conjugation we obtain $P E=e^{-\tau \psi\left(y, D_{x}\right)} \chi(x, y) e^{\tau \psi\left(y, D_{x}\right)}+R$. Taking a function $\chi_{0}(x, y) \in G_{0}^{\left(q_{1}, q_{2}\right)}(\Omega)$ such that $\chi_{0}(x, y)=1$ for $(x, y) \in \operatorname{supp}(\chi)$ and replacing $E$ by $E_{\chi}$, where $E_{\chi} u:=E(\chi(x, y) u)$, we have

$$
\begin{aligned}
P\left(x, y, D_{x}, D_{y}\right) E_{\chi} u & =e^{-\tau \psi\left(y, D_{x}\right)} \chi_{0}(x, y) e^{\tau \psi\left(y, D_{x}\right)} \chi(x, y) u+\bar{R} u \\
& =\chi(x, y) u-e^{-\tau \psi\left(y, D_{x}\right)} \widetilde{R}_{3} e^{\tau \psi\left(y, D_{x}\right)} u+\bar{R} u .
\end{aligned}
$$

The operator $\widetilde{R}_{3}=\left(1-\chi_{0}(x, y)\right) e^{\tau \psi\left(y, D_{x}\right)} \chi(x, y) e^{-\tau \psi\left(y, D_{x}\right)}$ is again regularizing on the anisotropic Sobolev spaces $H_{\frac{d}{m}}^{s}(\mathbb{R} \times(-\delta, \delta))$ in view of Proposition 3.1.

Remark 3.3. Theorem 3.3, Remark 3.2, and Theorem 3.1, combined with fixed point arguments as in Gramchev and Rodino [GR, Section 4], lead to the proofs of Theorem 1.1 and Theorem 1.2

Proof of Theorem 3.3. We limit ourselves for simplicity to proving the estimate (3.20) for $\alpha+\beta+\gamma+\theta=1$. The case $\alpha+\beta+\gamma+\theta>1$ does not involve actual complications; cf. Wakabayashi ([W], Theorem 2.6) or Kajitani and Wakabayashi ( $\mathrm{KW}$, Theorem 1.9) for the analytic frame. First we estimate the numerator of (3.20), and then we give some lemmas to estimate the denominator.

If $\alpha=1$, we get

$$
\begin{aligned}
& \left|D_{x} p(z, \zeta)\right| \lambda(\zeta)^{-\delta} \\
& \quad \leq\left|\sum_{(l, j) \in I} D_{x} h_{l j}(x, y) \eta^{j} \xi^{l}-D_{x} h_{d 0}(x, y) \xi^{d}\right| \lambda(\zeta)^{-\delta}+\left|D_{x} \sigma(z, \zeta)\right| \lambda(\zeta)^{-\delta} \\
& \quad \leq L_{1}\left(\left(\sum_{(l, j) \in I}|\eta|^{j} \xi^{l}+\xi^{d}\right) \lambda(\zeta)^{-\delta}+\lambda(\zeta)^{\bar{m}-\delta}\right) ;
\end{aligned}
$$

and for $\beta=1$,

$$
\left|D_{y} p(z, \zeta)\right| \lambda(\zeta)^{-\delta \frac{m}{d}} \leq L_{2}\left(\left(\sum_{(l, j) \in I}|\eta|^{j} \xi^{l}+\xi^{d}\right) \lambda(\zeta)^{-\delta \frac{m}{d}}+\lambda(\zeta)^{\bar{m}-\delta \frac{m}{d}}\right),
$$

for suitable constants $L_{1}, L_{2}$. If $\gamma=1$,

$$
\left|D_{\xi} p(z, \zeta)\right| \lambda(\zeta)^{\rho \frac{m}{d}} \leq L_{3}\left(\left(\sum_{(l, j) \in I}|\eta|^{j} \xi^{l-1}+\xi^{d-1}\right) \lambda(\zeta)^{\rho \frac{m}{d}}+\lambda(\zeta)^{\bar{m}-\frac{m}{d}(1-\rho)}\right) ;
$$

and for $\theta=1$,

$$
\left|D_{\eta} p(z, \zeta)\right| \lambda(\zeta)^{\rho} \leq L_{4}\left(\left(\sum_{(l, j) \in I}|\eta|^{j-1} \xi^{l}+|\eta|^{m-1}\right) \lambda(\zeta)^{\rho}+\lambda(\zeta)^{\bar{m}-(1-\rho)}\right),
$$

with suitable constants $L_{3}, L_{4}$.

Therefore, we observe that $\bar{m}-(1-\rho) \geq \bar{m}-\frac{m}{d}(1-\rho)$ since $d<m$, and $\bar{m}-(1-\rho)=\bar{m}-\delta>\bar{m}-\frac{m}{d} \delta$ since $\rho+\delta=1$. To prove (3.20), it will then be 
sufficient to show the boundedness, for $|\zeta|>B$, of the functions

$$
\begin{gathered}
Q_{1}(\zeta)=\frac{\left(\sum_{(l, j) \in I}|\eta|^{j} \xi^{l}+\xi^{d}\right) \lambda(\zeta)^{-\delta}}{|p(z, \zeta)|}, \\
Q_{2}(\zeta)=\frac{\left(|\eta|^{m-1}+\sum_{(l, j) \in I}|\eta|^{j-1} \xi^{l}\right) \lambda(\zeta)^{\rho}}{|p(z, \zeta)|}, \\
Q_{3}(\zeta)=\frac{\left(|\xi|^{d-1}+\sum_{(l, j) \in I}|\eta|^{j} \xi^{l-1}\right) \lambda(\zeta)^{\rho \frac{m}{d}}}{|p(z, \zeta)|}, \\
Q_{4}(\zeta)=\frac{\lambda(\zeta)^{\bar{m}-(1-\rho)}}{|p(z, \zeta)|}
\end{gathered}
$$

First we introduce three regions:

$$
\begin{aligned}
& R_{1}: c|\xi|^{d} \leq|\eta|^{m} \leq C|\xi|^{d}, \\
& R_{2}:|\eta|^{m} \geq C|\xi|^{d}, \\
& R_{3}:|\eta|^{m} \leq c|\xi|^{d}
\end{aligned}
$$

for suitable constants $c, C$ satisfying $c<\frac{1}{2} \min _{(x, y) \in \Omega}\left|\Re h_{d 0}(x, y)\right|$, and $C>$ $2 \max _{(x, y) \in \Omega}\left|\Re h_{d 0}(x, y)\right|$. We understand the neighborhood $\Lambda$ to be the region $R_{1}$.

The following inequalities then hold:

$$
\lambda(\zeta)^{-\delta} \leq\left\{\begin{array}{cll}
C^{\frac{\delta}{d}}|\eta|^{-\delta}, & \zeta \in R_{1}, & (I) \\
|\eta|^{-\delta}, & \zeta \in R_{2}, & (I I) \\
|\xi|^{-\delta \frac{d}{m}}, & \zeta \in R_{3}, & (I I I)
\end{array}\right.
$$

and

$$
\lambda(\zeta)^{\rho} \leq\left\{\begin{aligned}
C_{1}|\eta|^{\rho}, & \zeta \in R_{1}, \\
C_{2}|\eta|^{\rho}, & \zeta \in R_{2}, \\
C_{3}|\xi|^{\rho \frac{d}{m}}, & \zeta \in R_{3}
\end{aligned}\right.
$$

note that (II) and (III) in (3.25) hold for all $\zeta \in \mathbb{R}^{2}$, but for our aim we may limit ourselves to consider them respectively in $R_{2}$ and in $R_{3}$. By abuse of notation, in the following we shall also denote by $R_{1}, R_{2}, R_{3}$ the sets $\Omega \times R_{1}, \Omega \times R_{2}, \Omega \times R_{3}$; recall that $\Gamma=\Omega \times \Lambda$.

Lemma 3.1. Let $p(z, \zeta)$ be the function (3.16), such that (i),(ii),(iii) in (3.18) hold. Then there are positive constants $K_{1}<1$ and $B$, such that

$$
|p(z, \zeta)| \geq K_{1}\left|\Im h_{l^{*} j^{*}}(x, y)\right||\eta|^{j^{*}}|\xi|^{l^{*}}, \quad(z, \zeta) \in R_{1},|\zeta|>B .
$$

Proof. We have

$$
\begin{aligned}
|p(z, \zeta)|^{2} & =\left(\eta^{m}-\Re h_{d 0}(x, y) \xi^{d}+\sum_{(l, j) \in I} \Re h_{l j}(x, y) \eta^{j} \xi^{l}\right. \\
& +\Re \sigma(z, \zeta))^{2}+\left(\Im h_{l^{*} j^{*}}(x, y) \eta^{j^{*}} \xi^{l^{*}}\right. \\
& +\sum_{(l, j) \in I_{-}} \Im h_{l j}(x, y) \eta^{j} \xi^{l}+\sum_{(l, j) \in I_{+}} \Im h_{l j}(x, y) \eta^{j} \xi^{l} \\
& \left.-\Im h_{d 0}(x, y) \xi^{d}+\Im \sigma(z, \zeta)\right)^{2} .
\end{aligned}
$$


By removing the terms arising from the real part of $p(z, \zeta)$, we can write

$$
|p(z, \zeta)|^{2} \geq \Im h_{l^{*} j^{*}}(x, y)^{2} \eta^{2 j^{*}} \xi^{2 l^{*}}+\sum_{i=1}^{5} J_{i}(z, \zeta),
$$

where

$$
\begin{gathered}
J_{1}=\left(\sum_{(l, j) \in I_{-}} \Im h_{l j}(z) \eta^{j} \xi^{l}+\sum_{(l, j) \in I_{+}} \Im h_{l j}(z) \eta^{j} \xi^{l}-\Im h_{d 0}(z) \xi^{d}+\Im \sigma(z, \zeta)\right)^{2}, \\
J_{2}(z, \zeta)=2 \Im h_{l^{*} j^{*}}(x, y) \sum_{(l, j) \in I_{-}} \Im h_{l j}(x, y) \eta^{j^{*}+j} \xi^{l^{*}+l}, \\
J_{3}(z, \zeta)=2 \Im h_{l^{*} j^{*}}(x, y) \sum_{(l, j) \in I_{+}} \Im h_{l j}(x, y) \eta^{j^{*}+j} \xi^{l^{*}+l}, \\
J_{4}(z, \zeta)=-2 \Im h_{l^{*} j^{*}}(x, y) \Im h_{d 0}(x, y) \eta^{j^{*}} \xi^{l^{*}+d}, \\
J_{5}(z, \zeta)=2 \Im \sigma(z, \zeta) \Im h_{l^{*} j^{*}}(x, y) .
\end{gathered}
$$

The function (3.28) is nonnegative; (3.30) and (3.31) are also nonnegative by hypotheses $($ ii $),($ iii $)$. Let us fix attention on $J_{2}(z, \zeta)$, defined by (3.29). We have, for all $\epsilon>0$,

$$
\left(\Im h_{l^{*} j^{*}}(x, y)\right)^{2} \eta^{2 j^{*}} \xi^{2 l^{*}}+J_{2}(z, \zeta) \geq(1-\epsilon)\left(\Im h_{l^{*} j^{*}}(x, y)\right)^{2} \eta^{2 j^{*}} \xi^{2 l^{*}},
$$

in $R_{1},|\zeta|>B$. In fact, by (3.24) in $R_{1}$ and hypothesis $(i)$ in (3.18), for all $\epsilon>0$ we get, for $B$ sufficiently large,

$$
\begin{aligned}
\frac{\left|J_{2}(z, \zeta)\right|}{\left(\Im h_{l^{*} j^{*}}(x, y)\right)^{2} \eta^{2 j^{*}} \xi^{2 l^{*}}} & \leq \mathrm{const} \sum_{(l, j) \in I_{-}} \frac{|\eta|^{j^{*}+j}|\xi|^{l^{*}+l}}{\eta^{2 j^{*}} \xi^{2 l^{*}}} \\
& \leq \mathrm{const} \sum_{(l, j) \in I_{-}} \frac{|\eta|^{j^{*}+j+\left(l^{*}+l\right) \frac{m}{d}}}{\eta^{2 j^{*}+2 l^{*} \frac{m}{d}}}<\epsilon, \quad|\zeta|>B .
\end{aligned}
$$

We remark that $k^{*}=d j^{*}+m l^{*}>k^{-}=d j+m l$.

Concerning (3.32), since $\bar{m}<\frac{k^{*}}{d}$, we have

$$
\left(\Im h_{l^{*} j^{*}}(x, y)\right)^{2} \eta^{2 j^{*}} \xi^{2 l^{*}}+J_{5}(z, \zeta) \geq(1-\epsilon)\left(\Im h_{l^{*} j^{*}}(x, y)\right)^{2} \eta^{2 j^{*}} \xi^{2 l^{*}}
$$

Then

$$
|p(z, \zeta)| \geq K_{1}\left|\Im h_{l^{*} j^{*}}(x, y)\right||\eta|^{j^{*}}|\xi|^{*^{*}}, \quad(z, \zeta) \in R_{1},|\zeta|>B
$$

for a suitable positive constant $K_{1}$.

Lemma 3.2. Let $p(z, \zeta)$ be the function (3.16). Then there are positive constants $K_{2}<1$ and $B$, such that

$$
|p(z, \zeta)| \geq K_{2}|\eta|^{m}, \quad(z, \zeta) \in R_{2},|\zeta|>B .
$$


Proof. We write $|p(z, \zeta)|^{2}$ as in (3.27); by removing the terms arising from the imaginary part of $p(z, \zeta)$, we get

$$
|p(z, \zeta)|^{2} \geq\left(\eta^{m}-\Re h_{d 0}(x, y) \xi^{d}\right)^{2}+W_{1}(z, \zeta)+W_{2}(z, \zeta)+W_{3}(z, \zeta),
$$

where

$$
\begin{aligned}
W_{1}(z, \zeta)= & \left(\sum_{(l, j) \in I} \Re h_{l j}(x, y) \eta^{j} \xi^{l}+\Re \sigma(z, \zeta)\right)^{2}, \\
W_{2}(z, \zeta)=2 & \sum_{(l, j) \in I} \Re h_{l j}(x, y) \eta^{j+m} \xi^{l} \\
& -2 \Re h_{d 0}(x, y) \sum_{(l, j) \in I} \Re h_{l j}(x, y) \eta^{j} \xi^{l+d} . \\
W_{3}(z, \zeta)= & 2 \eta^{m} \Re \sigma(z, \zeta)-2 \Re h_{d 0}(x, y) \xi^{d} \sigma(z, \zeta) .
\end{aligned}
$$

Observe first that, for $\lambda>0$ sufficiently small,

$$
\left(\eta^{m}-\Re h_{d 0}(x, y) \xi^{d}\right)^{2}>\lambda \eta^{2 m} .
$$

In fact,

$$
\left(\eta^{m}-\Re h_{d 0}(x, y) \xi^{d}\right)^{2} \geq \eta^{2 m}-2 \Re h_{d 0}(x, y) \eta^{m} \xi^{d},
$$

and using (3.24) in $R_{2}$, we have

$$
\eta^{2 m}-2 \Re h_{d 0}(x, y) \eta^{m} \xi^{d} \geq\left(1-\frac{2}{C} \Re h_{d 0}(x, y)\right) \eta^{2 m}>\lambda \eta^{2 m},
$$

since $C>2 \max _{(x, y) \in \Omega}\left|\Re h_{d 0}(x, y)\right|$.

Equation (3.35) is nonnegative. We denote (3.36) by $\Upsilon_{1}(z, \zeta)-\Upsilon_{2}(z, \zeta)$ and (3.37) by $\Upsilon_{3}(z, \zeta)-\Upsilon_{4}(z, \zeta)$. Then

$$
|p(z, \zeta)|^{2} \geq \lambda \eta^{2 m}+\Upsilon_{1}(z, \zeta)-\Upsilon_{2}(z, \zeta)+\Upsilon_{3}(z, \zeta)-\Upsilon_{4}(z, \zeta) .
$$

Arguing on $\Upsilon_{1}-\Upsilon_{2}, \Upsilon_{3}-\Upsilon_{4}$ in the same way as we did in Lemma 3.1, we can show that for all $\epsilon>0$,

$$
\lambda \eta^{2 m}+\Upsilon_{1}(z, \zeta)-\Upsilon_{2}(z, \zeta) \geq(\lambda-\epsilon) \eta^{2 m}, \quad(z, \zeta) \in R_{2},|\zeta|>B
$$

and

$$
\lambda \eta^{2 m}+\Upsilon_{3}(z, \zeta)-\Upsilon_{4}(z, \zeta) \geq(\lambda-\epsilon) \eta^{2 m}, \quad(z, \zeta) \in R_{2},|\zeta|>B .
$$

Thus

$$
|p(z, \zeta)| \geq K_{2}|\eta|^{m}, \quad(z, \zeta) \in R_{2},|\zeta|>B
$$

where $K_{2}=(\lambda-\epsilon)^{\frac{1}{2}}$.

Lemma 3.3. Let $p(z, \zeta)$ be the function (3.16), such that (iv) in (3.18) holds. Then there are positive constants $K_{3}<1$ and $B$ such that

$$
|p(z, \zeta)| \geq K_{3}|\xi|^{d}, \quad(z, \zeta) \in R_{3},|\zeta|>B .
$$


Proof. Again we apply (3.34), (3.35), (3.36), (3.37) to $|p(z, \zeta)|^{2}$. Observe that in $R_{3}$, arguing as above, since $c<\frac{1}{2} \min _{(x, y) \in \Omega}\left|\Re h_{d 0}(x, y)\right|$, we obtain, for a suitable constant $\mu>0$,

$$
\left(\eta^{m}-\Re h_{d 0}(x, y) \xi^{d}\right)^{2}>\mu \xi^{2 d} .
$$

About the terms in (3.35), (3.36) and (3.37), the remarks we made in Lemma 3.2 hold on replacing $\lambda \eta^{2 m}$ with $\mu \xi^{2 d}$. Then we have

$$
|p(z, \zeta)| \geq K_{3}|\xi|^{d}, \quad(z, \zeta) \in R_{3},|\zeta|>B,
$$

where $K_{3}=(\mu-\epsilon)^{\frac{1}{2}}$.

We first consider $Q_{1}(\zeta)$ separately in the regions $R_{1}, R_{2}, R_{3}$, to prove boundedness. In $R_{1}$ by (3.25), (3.26) we get easily, writing as before $k=d j+m l$,

$$
Q_{1}(\zeta) \leq \operatorname{const}\left(\sum_{k} \frac{1}{|\eta|^{m-\frac{k}{d}}}+1\right),|\zeta|>B,
$$

where $m-\frac{k}{d}>0$ by definition of $I$ and $I_{k}$. In the region $R_{2}$ we have $|p(z, \zeta)| \geq$ $|\eta|^{m}>|\eta|^{\frac{k^{*}}{d}}$. In $R_{3}$, by using (3.25) and (3.38) for a constant $\epsilon>0$ which we may take as small as we want by fixing $B$ sufficiently large, we have

$$
Q_{1}(\zeta) \leq \operatorname{const}\left(\sum_{k} \frac{1}{|\xi|^{2 d-\frac{k}{m}-\frac{k^{*}}{m}}}+\frac{1}{|\xi|^{\delta \frac{d}{m}}}\right)<\epsilon,|\zeta|>B .
$$

We have therefore proved that $Q_{1}(\zeta)$ is bounded. Arguing in the same way on $Q_{2}(\zeta), Q_{3}(\zeta)$ and $Q_{4}(\zeta)$, we prove their boundedness in $\mathbb{R}^{2}$.

Remark 3.4. By formulas (3.26), (3.33), (3.38), we obtain that $|p(z, \zeta)| \geq a|\zeta|^{\frac{k^{*}}{m}}$, $a>0,|\zeta|>B$, since we are considering the case when $d j+m l<d m$. If we refer to the anisotropic weight function $\lambda(\zeta)=|\xi|^{\frac{d}{m}}+|\eta| \sim\left(|\xi|^{d}+|\eta|^{m}\right)^{\frac{1}{m}}$, we find that

$$
|p(z, \zeta)| \geq b \lambda(\zeta)^{\frac{k^{*}}{d}},|\zeta|>B
$$

for a suitable positive constant $b$.

Now Lemma 3.1. Lemma 3.2. Lemma 3.3 and the estimate (3.39) complete the proof.

Remark 3.5. It is possible to propose a geometric invariant generalization of Theorems 1.1 and 1.2 to pseudo-differential operators with involutive characteristics of multiplicity $m \geq 4$, in more than two space variables, by arguing microlocally, using classical Fourier integral operators and $S_{\rho, \delta}^{m}$ arguments. This will be the subject of another paper. 


\section{ACKNOWLEDGEMENT}

The authors thank the unknown referee for critical remarks which led to improvements in the paper.

\section{REFERENCES}

[BT] A. Bove and D. Tartakoff, Propagation of Gevrey regularity for a class of hypoelliptic equations, Trans. Amer. Math. Soc., 348 (1996), 2533-2575. MR 96i:35017

[BC] J. M. Bony and J. Y. Chemin, Espaces fonctionnels associés au calcul de WeylHörmander, Bull. Soc. Math. France, 122 (1994), 77-118. MR 95a:35152

[CZ] M. Cicognani and L. Zanghirati, On a class of unsolvable operators, Ann. Scuola Norm. Sup. Pisa Cl. Sci. (4), 20 (1993), 357-369. MR 95d:35003

[C] A. Corli, On local solvability of linear partial differential operators with multiple characteristics, J. Differential Equations, 81 (1989), 275-293. MR 91e:35007

[C2] A. Corli, On local solvability in Gevrey classes of linear partial differential operators with multiple characteristics, Comm. Partial Differential Equations, 14 (1989), 1-25. MR 90a:35001

[DR2] G. De Donno and L. Rodino, Gevrey hypoellipticity for partial differential equations with characteristics of higher multiplicity, to appear in Rend. Sem. Mat. Univ. Politecnico Torino, (2000).

[DR3] G. De Donno and L. Rodino, Gevrey hypoellipticity for equations with involutive characteristics of higher multiplicity, C. R. Acad. Bulgare Sci., 53 No. 7, (2000), 25-30. MR 2001g:35045

[D] B. Dehman, Résolubilité local pour des équations semi-linéaires complexes, Canad. J. Math., 42 (1990), 126-140. MR 91h:35008

[ES] Y. V. Egorov and D. W. Schulze, Pseudo-differential operators, singularities, applications, Operator Theory: Advances and Applications, Vol. 93, Birkhäuser-Verlag, Basel-Boston-Berlin, 1997. MR 98e:35181

[G] G. Garello, Inhomogeneuos paramultiplication and microlocal singularities for semilinear equations, Boll. Un. Mat. Ital. (7) 10-B (1996), 885-902. MR 97k:35007

[G1] G. Garello, Local solvability for semilinear equations with multiple characteristics, Ann. Univ. Ferrara Sez. VII, Sci. Mat. 41 (1996), suppl., 199-209. MR 98i:35003

[GG] T. Gramchev, On the critical index of Gevrey solvability for some linear partial differential equations, Workshop on Partial Differential Equations (Ferrara 1999), Ann. Univ. Ferrara Sez. VII (N.S.), 45 (1999), suppl. (2000), 139-153. MR 2002f:35006

[GP] T. Gramchev and P. Popivanov, Local solvability of semilinear partial differential equations, Ann. Univ. Ferrara Sez. VII - Sc. Mat. 35 (1989), 147-154. MR 91m:35006

[GP1] T. Gramchev and P. Popivanov, Partial differential equations: Approximate solutions in scales of functional spaces, Mathematical Research, 108, Wiley-VCH Verlag, Berlin, 2000. MR 2001g:35002

[GPY] T. Gramchev, P. Popivanov, and M. Yoshino, Critical Gevrey index for hypoellipticity of parabolic operators and Newton polygons, Ann. Mat. Pura Appl., 170 (1996), 103131. MR 98c:35029

[GR] T. Gramchev and L. Rodino, Gevrey solvability for semilinear partial differential equations with multiple characteristics, Boll. Un. Mat. Ital. B (8), 2 (1999), 65-120. MR 2001j:35006

$[\mathrm{H}]$ L. Hörmander, The analysis of linear partial differential operators, I, II, III, IV, Grundlehren der Mathematischen Wissenschaften, Springer-Verlag, 1983-1985, Berlin. MR 85g:35002a MR 85g:35002b MR 87d:35002a MR 87d:35002b

[HS] J. Hounie and P. Santiago, On the local solvability of semilinear equations, Comm. in Partial Differential Equations, 20 (1995), 1777-1789. MR 96h:35005

[HP] C. Hunt and A. Piriou, Opérateurs pseudo-différentiels anisotropes d'ordre variable, C. R. Acad. Sci. Paris, 268 (1969), 28-31. MR 40:1819

[HP1] C. Hunt and A. Piriou, Majorations $L^{2}$ et inégalité sous-elliptique pour les opérateurs pseudo-différentiels anisotropes d'ordre variable, C. R. Acad. Sci. Paris, 268 (1969), 214-217. MR 40:1820 
[KS] K. Kajitani and S. Spagnolo, in progress, communicated to the meeting "Perturbative methods for nonlinear partial differential equations", Cagliari 2000.

[KW] K. Kajitani and S. Wakabayashi, Hypoelliptic operators in Gevrey classes, in "Recent developments in hyperbolic equations" L. Cattabriga, F. Colombini, M.K.V. Murthy, S. Spagnolo, editors, Longman 1988, London, 115-134. MR 90e:35041

[LA] R. Lascar, Distributions intégrale de Fourier et classes de Denjoy-Carleman. Applications, C. R. Acad Sci. Paris, Sér. A 284, (1977), 485-488. MR 55:906

[L] H. Lewy, An example of a smooth linear partial differential equation without solution, Ann. of Math. (2), 66 (1957), 155-158. MR 19:551d

[LR1] O. Liess and L. Rodino, Inhomogeneous Gevrey classes and related pseudo-differential operators, Boll. Un. Mat. Ital., Sez. IV, 3-C (1984), 233-323. MR 85k:35239

[LR2] O. Liess and L. Rodino, Linear partial differential equations with multiple involutive characteristics, in "Microlocal analysis and spectral theory", L. Rodino, editor, Kluwer, 1997, Dordrecht, 1-38. MR 98e:35034

[LO] M. Lorenz, Anisotropic operators with characteristics of constant multiplicity, Math. Nachr., 124 (1985), 199-216. MR 87f:35249

[MA] P. Marcolongo, Solvability and nonsolvability for partial differential equations in Gevrey spaces, Ph.D. Dissertation, Mathematics, University of Torino, 2000.

[MO] P. Marcolongo and A. Oliaro, Local solvability for semilinear anisotropic partial differential equations, Ann. Mat. Pura e Appl. (4) 170 (2001), 229-262. MR 2002h:35004

[MR] M. Mascarello and L. Rodino, Partial differential equations with multiple characteristics, Wiley-VCH, 1997, Berlin. MR 99a:35009

[M] A. Menikoff, On hypoelliptic operators with double characteristics, Ann. Scuola Norm. Sup. Pisa, Cl. Sci. (4) 4 (1977), 689-724. MR 57:13156

[P1] P. R. Popivanov, On the local solvability of a certain class of pseudo-differential equations with double characteristics, Trudy Sem. Petrovsk., 1 (1975), 237-278; Amer. Math. Soc. Transl., 118 (1982), 51-90. MR 55:841

[P2] P. R. Popivanov, Local solvability of some classes of linear differential operators with multiple characteristics, Ann. Univ. Ferrara, Seg. VII, Sci. Mat. 45 suppl. (1999), 263274. MR 2001j:35038

[P3] P. R. Popivanov, Microlocal properties of a class of pseudodifferential operators with double involutive characteristics, Partial Differential Equations, Banach Center Publications, Volume 19, PWN-Polish Scientific Publishers, Warsaw, 1987, pp. 213-224. MR 91i:35221

[PP] P. R. Popivanov and G. S. Popov, Microlocal properties of a class of pseudo-differential operators with multiple characteristics, Serdica, 6 (1980), 167-181. (Russian) MR 82d:35038

[R] G. B. Roberts, Quasi-subelliptic estimates for operators with multiple characteristics, Comm. Partial Differential Equations, 11 (1986), 231-320. MR 87e:35024

[RO] L. Rodino, Linear partial differential operators in Gevrey spaces, World Scientific Publishing Co., River Edge, NJ, 1993. MR 95c:35001

[RO2] L. Rodino, Local solvability in Gevrey classes, in: Hyperbolic Equations (Padua 1985), 167-185, Pitman Research Notes in Math. Ser., 158, Longman, Harlow, 1987. MR 89d:35001

[S] N. A. Šananin, The local solvability of equations of quasi-principal type, Mat. Sb. (N.S.) 97 (139), (1975), 503-516; English transl., Math. USSR Sb. 26 (1975), 458-470. MR 57:13112

[SE] F. Segàla, A class of locally solvable differential operators, Boll. Un. Mat. Ital. B (6), 4 (1985), 241-251. MR 86i:35003

[SP] S. Spagnolo, Local and semi-global solvability for systems of non-principal type, Comm. Partial Differential Equations, 25, no. 5-6, (2000), 1115-1141. MR 2002d:35231

[T] F. Trèves, Introduction to pseudodifferential and Fourier integral operators. I, II, The University Series in Mathematics, Plenum Press, 1980, New York and London. MR 82i:35173; MR 82i:58068

[TU] V. N. Tulovsky, Propagation of singularities of operators with characteristics of constant multiplicity, Trudy Moskov. Mat. Obshch., 39 (1979), 113-134; English transl., Trans. Moscow Math. Soc., 1981, no. 1 (39), 121-144. MR 82m:35150 
[W] S. Wakabayashi, Singularities of solutions of the Cauchy problem for hyperbolic systems in Gevrey classes, Japan J. Math., 11 (1985), 157-201. MR 88h:35067

Dipartimento di Matematica, Università di Torino, Via Carlo Alberto 10, 10123 TORINO, ITALY

E-mail address: dedonno@dm.unito.it

Dipartimento di Matematica, Università di Torino, Via Carlo Alberto 10, 10123 TORINO, ITALY

E-mail address: oliaro@dm.unito.it 